
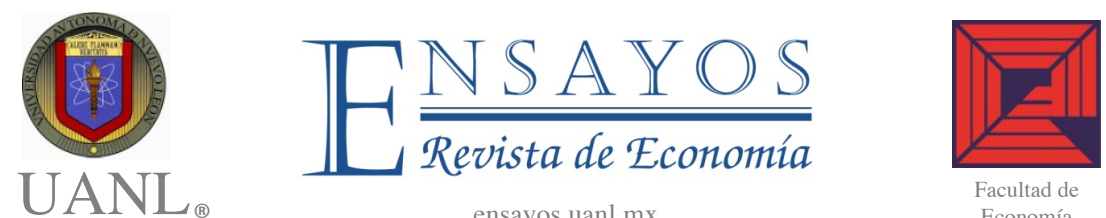

\title{
Sensibilidad y asimetrías ante choques de ingreso en el consumo privado de México, 1995-2017
}

\section{Sensitivity and asymmetries of income shocks in Mexico's private consumption, 1995-2017}

Miguel Ángel Mendoza González

\begin{tabular}{lll}
\hline $\begin{array}{l}\text { Información del } \\
\text { artículo }\end{array}$ & Resumen \\
\cline { 1 - 2 } $\begin{array}{l}\text { Recibido: } \\
\text { 17 septiembre 2018 }\end{array}$ & $\begin{array}{l}\text { Esta investigación analiza con un enfoque poskeynesiano } \\
\text { la sobre-sensibilidad y las asimetrías ante choques de } \\
\text { ingreso del consumo privado y su desagregación en bienes } \\
\text { duraderos, semi-duraderos, no-duraderos y los servicios en }\end{array}$ \\
$\begin{array}{ll}\text { Aceptado: } \\
19 \text { febrero 2020 }\end{array}$ & $\begin{array}{l}\text { México para el período 1995-2017. Para estimar la sobre- } \\
\text { sensibilidad se utilizan las metodologías de cointegración } \\
\text { de Johansen-Juselius y el método de estimación FMOLS, }\end{array}$ \\
$\begin{array}{l}\text { Clasificación JEL: C50; } \\
\text { D12; E12; E21; F62; J61 }\end{array}$ & $\begin{array}{l}\text { sin sesgo de endogeneidad de Phillips y Hansen. Para las } \\
\text { asimetrías se aplican las pruebas de Seo y Hansen-Seo de } \\
\text { cointegración, con regímenes múltiples (cointegración- } \\
\text { threshold) y modelos TVECM de vectores de corrección de }\end{array}$ \\
$\begin{array}{l}\text { Sobre-sensibilidad del } \\
\text { consumo; Asimetrías a } \\
\text { choques de ingreso; }\end{array}$ & $\begin{array}{l}\text { error, con regímenes múltiples de Seo. Con los resultados } \\
\text { se comprueba la existencia de sobre-sensibilidad del }\end{array}$ \\
$\begin{array}{l}\text { Cointegración con } \\
\text { regímenes múltiples; } \\
\text { Modelos } V E C M \text { con }\end{array}$ & $\begin{array}{l}\text { consumo privado y del consumo de la mayoría de los } \\
\text { bienes por durabilidad (nacional e importado) y } \\
\text { regímenes múltiples; } \\
T V E C M\end{array}$ & $\begin{array}{l}\text { comportamientos asimétricos de régimen bajo, consistente } \\
\text { con un escenario de crisis económica. }\end{array}$ \\
\hline
\end{tabular}

*Facultad de Economía, UNAM; email: mendozag@unam.mx

ISSN Electrónico: 2448-8402 | ISSN Impreso: 1870-221X | (C2020 Los autores @) () 


\begin{tabular}{|c|c|}
\hline Article information & Abstract \\
\hline $\begin{array}{l}\text { Received: } \\
17 \text { September } 2018 \\
\text { Accepted: } \\
19 \text { February } 2020\end{array}$ & $\begin{array}{l}\text { With a Postkeynesian approach, this research analyzes the } \\
\text { over-sensitivity and asymmetries of income shocks of } \\
\text { private consumption and its unbundling into durable, } \\
\text { semi-durable, non-durable goods and services in Mexico } \\
\text { for the period 1995-2017. To estimate over-sensitivity to }\end{array}$ \\
\hline $\begin{array}{l}\text { JEL Classification: } \\
\text { C50; D12; E12; E21; } \\
\text { F62; J61 }\end{array}$ & $\begin{array}{l}\text { income, cointegration methodologies from Johansen- } \\
\text { Juselius are used and the FMOLS estimation method } \\
\text { without endogeneity bias of Phillips and Hansen. And, for } \\
\text { the asymmetries, this study applies the tests of Seo and }\end{array}$ \\
\hline $\begin{array}{l}\text { Keywords: } \\
\text { over-sensitivity of } \\
\text { consumption; } \\
\text { asymmetries to income } \\
\text { shocks; threshold- } \\
\text { cointegration; threshold- } \\
\text { VECM models; TVECM }\end{array}$ & $\begin{array}{l}\text { Hansen-Seo of cointegration with multiple regimes } \\
\text { (threshold-cointegration) and threshold vector error } \\
\text { correction model (threshold-VECM) with multiple } \\
\text { regimes of Seo. The results show the existence of over- } \\
\text { sensitivity of private consumption and durability for most } \\
\text { of the goods (national and imported) and low-level } \\
\text { asymmetric behaviors consistent with a scenario of } \\
\text { economic crisis. }\end{array}$ \\
\hline
\end{tabular}

\section{Introducción}

La hipótesis del ingreso absoluto establece que el elemento más importante de la explicación del consumo privado es el ingreso corriente, con propensiones marginales y medias que en combinación generan una elasticidad ingreso igual o menor a la unidad. Algunos planteamientos poskeynesianos establecen que el ingreso corriente es el principal factor explicativo del consumo y que, en segundo plano, influyen factores como el ahorro, los créditos, las tasas de interés, los precios relativos y el tipo de cambio real (Lavoie, 1994; Lavoie, 1994b); desde el punto de vista empírico, lo esperado serían elasticidades ingresos mayores a las de los otros factores explicativos. En el otro sentido, con la teoría del ingreso permanente, Friedman (1957) plantea que el consumo no depende del ingreso corriente sino de los ingresos permanentes y transitorios, y de este último se ha identificado lo que se llama la "sensibilidad excesiva" del consumo al ingreso transitorio. Campbell y Mankiw (1989) explican que esta se presenta cuando los hogares tienen problemas de liquidez y su patrón de consumo se vuelve más sensible a modificaciones del ingreso; sobre todo en periodos de crisis económicas.

En nuestra investigación, proponemos el concepto de "sobre-sensibilidad" del consumo a los choques de ingreso con fundamentos poskeynesianos, que se identifica por una elasticidad ingreso corriente de largo plazo mayor a la unidad, combinada con una propensión marginal a consumir mayor a 0.8 , que se relacionan con dos escenarios importantes: el primero es cuando la problemática se vincula a la disponibilidad de financiamiento y en especial del consumo (Blundell, et al., 1992); y el segundo, cuando los consumidores consideran cambios de precios relativos o del tipo de cambio real y comparan 
los precios de productos nacionales e importados (Lavoie, 1994; Lavoie, 1994b). Por otro lado, la mayoría de las investigaciones empíricas sobre México suponen que el consumo y el ingreso tienen una relación de equilibrio, con un comportamiento estable de las propensiones media y marginal, y de la elasticidad ingreso del consumo de largo plazo (Castro, et al., 1997; RuizGalindo y Venegas-Martínez, 2007; Mendoza-González, 2020). Sin embargo, las discusiones sobre regímenes de los comportamientos macroeconómicos tasas de interés, demanda monetaria, desigualdad económica, etc-, tienen claras implicaciones sobre la posibilidad de relaciones asimétricas entre el consumo y el ingreso corriente (Bunn, et al., 2017; Ludwig, 2015). En términos del análisis econométrico, el planteamiento de las asimetrías considera que los desequilibrios de corto plazo pueden tomar diferentes rutas, distancias y tiempos frente al equilibrio definido por el vector de cointegración (Hansen y Seo, 2002; Seo, 2006; Seo, 2011).

El objetivo principal de esta investigación fue el analizar las sensibilidades y las asimetrías en choques de ingreso sobre el consumo privado y su desagregación en México desde una perspectiva poskeynesiana, donde el consumo se determina por el ingreso corriente, el crédito del consumo y los precios relativos en largo plazo. Para el análisis de la desagregación del consumo privado se consideraron los bienes duraderos, semi-duraderos, noduraderos nacionales e importados y de los servicios en México, para el periodo de 1995-2017. La metodología que se propone, utiliza relaciones de cointegración para medir las elasticidades ingreso corriente de equilibrio de largo plazo, con diferentes bienes del consumo: nacionales, importados y servicios. Para obtener las elasticidades ingreso corriente de la estimación de vectores de cointegración, con variables exógenas adicionales como el crédito del consumo y el tipo de cambio real, para diferentes equilibrios de largo plazo. Con tales elasticidades ingreso corriente de equilibrio de largo plazo, se analizan la posibilidad de asimetrías de los choques de ingreso de corto plazo con las pruebas de Seo (2006) y Hansen y Seo (2002) de no cointegración, cointegración lineal y cointegración con regímenes múltiples (threshold); para medir las asimetrías de corto plazo se estiman modelos de Vectores de Corrección de Error (VECM) con regímenes múltiples en ajustes de corto plazo (TVECM); y, para analizar la sensibilidad a los choques de ingreso corriente, los modelos TVECM se condicionan con tres posibilidades de elasticidades ingreso de largo plazo.

Este documento consta de: esta introducción; el apartado uno con los aspectos teóricos y analíticos sobre los determinantes del consumo; el apartado dos, donde se presentan las metodologías de cointegración de Johansen y Juselius (1990) y el método de estimación FMOLS sin sesgo de endogeneidad (Phillips \& Hansen, 1990; Spanos, 2003) para estimar funciones de consumo con factores explicativos poskeynesianos: ingreso corriente, el crédito del consumo 
y el tipo de cambio real. Las pruebas de Seo (2006), Hansen y Seo (2002), y la especificación y estimación de los modelos TVECM (Seo, 2011), se utilizan para identificar asimetrías de corto plazo; la parte tres analiza los resultados de la aplicación de la metodología; al final, se presentan las conclusiones generales y la bibliografía.

\section{Aspectos analíticos y empíricos sobre la sensibilidad y comportamientos asimétricos del consumo, choques de ingreso}

\subsection{La sensibilidad al ingreso y los comportamientos asimétricos}

La hipótesis del ingreso absoluto establece que la principal fuente de decisión de los consumidores es el ingreso corriente, por lo que la tendencia a cero (uno) de la propensión marginal a consumir implica que el consumo es menos (más) sensible a modificaciones del ingreso. En tanto, la elasticidad del ingreso corriente del consumo que mide la sensibilidad relativa, comienza en cero y puede alcanzar valores mayores a la unidad, de acuerdo con la relación entre la propensión marginal y la propensión media. Cuando la propensión marginal es menor (mayor) a la propensión media, la elasticidad ingreso será menor (mayor) a la unidad; esto implica que cuando se observa una elasticidad unitaria del ingreso significa que las propensiones marginales y media son iguales. La hipótesis de la "sensibilidad excesiva" del ingreso es una regularidad empírica que se deriva de la hipótesis del ingreso permanente (Friedman, 1957), y establece que el ingreso se divide en permanente y transitoria, por lo que los choques de ingreso se suponen no esperados (Jappelli \& Pistaferri, 2010) y transitorios (Bayoumi \& Sgherri, 2007), y estos últimos provocan un estado de sobre-reacción de los consumidores sobre todo cuando las propensiones marginales y medias son especialmente altas (mayor a 0.8 ); la tendencia de la elasticidad ingreso transitorio es mayor a la unidad cuando la propensión marginal es relativamente mayor a la propensión media.

En el trabajo de Campbell y Mankiw (1989), se explica que la razón fundamental de esta sensibilidad excesiva son consumidores con problemas de liquidez por su alta propensión marginal a consumir, por lo que cualquier modificación (choque) en los ingresos, en particular en periodos de crisis económicas, modifica sensiblemente el patrón del consumo. En el análisis macroeconómico, las restricciones de liquidez se pueden reducir con una política de liberalización financiera con el aumento de las disposiciones de créditos del consumo (Blundell, et al., 1992). El otro aspecto que disminuye los efectos de la sensibilidad excesiva en las decisiones de los consumidores, son los precios relativos y en especial el tipo de cambio real. Aunque son los micro-fundamentos neoclásicos los que establecen la importancia de los precios relativos en la determinación del patrón del consumo, en la postura 
poskeynesiana (Lavoie, 1994; Lavoie, 1994b) prevalece la importancia del ingreso corriente y en segundo lugar pueden influir los precios relativos, junto con otros factores explicativos como el ahorro, los créditos, tasas de interés, etc.

En la investigación de Bunn, et al. (2017), se presentan tres predicciones teóricas ligadas a respuestas asimétricas, que igual son consistentes para analizar choques de ingreso corriente en el consumo en una perspectiva poskeynesiana. La primera predicción muestra que cuando los hogares tienen una alta propensión marginal a consumir y acceso imperfecto al mercado de crédito, reducen el consumo más rápido a un choque de ingreso negativo $\mathrm{y}$ ajustan lento su nuevo patrón del consumo ante un choque de ingreso positivo. La siguiente predicción es cuando los hogares responden diferente a los choques de ingresos positivos y negativos, dependiendo de su disponibilidad de ahorro. La experiencia de los hogares condiciona una reacción más rápida a situaciones de choques de ingreso negativos, por lo que tienden a disponer de mayores recursos provenientes del ahorro para suavizar el efecto sobre su patrón del consumo. En tanto, el patrón del consumo se ajusta lento y en menor medida, hasta que exista un choque de ingreso positivo, por lo que la tendencia del ahorro es creciente. La última predicción proviene del conocimiento o experiencia de los hogares sobre la ponderación de la observación de la mayor proporción de perdedores, con respecto a ganadores, en los periodos de crecimiento y de crisis económicas, que provoca una respuesta asimétrica del consumo a choques de ingreso corriente.

\subsection{Estudios empíricos sobre choques de ingreso}

En el estudio de Jappelli y Pistaferri (2010) se propone un esquema analítico para entender cómo el consumo responde a los choques de ingreso. Ellos establecen que los consumidores responden diferente, dependiendo de si los cambios del ingreso son o no anticipados. Los cambios anticipados pueden suponer ingresos crecientes o decrecientes, pero en los primeros se pueden anticipar cambios pequeños y grandes, mientras que en los cambios anticipados de ingresos menores, la magnitud no es relevante. En el caso de cambios del ingreso no-anticipados, se pueden dividir en choques permanentes $\mathrm{y}$ transitorios, $\mathrm{y}$ estos últimos pueden ser positivos o negativos. También elaboraron un resumen sobre los diferentes enfoques de las pruebas de hipótesis y, para organizar su discusión, especifican el crecimiento del consumo $\quad \Delta l n c_{i t}=z^{\prime}{ }_{i t} \lambda+\alpha E_{t-1} \Delta \ln y_{i t}+\sum_{k=1}^{K} \varphi^{k} \pi_{i t}^{k}+\xi_{i t}$, donde las variables $z^{\prime}{ }_{i t}$ capturan los efectos de cambios de las preferencias, demográficos, ahorro precautorio, etc., sobre el crecimiento del consumo, y $\xi_{i t}$ es el error que puede incluir errores de medición en el consumo. Con tal especificación, establecen hipótesis generales como suponer que los cambios 
esperados del ingreso no afectan el consumo $(\alpha=0) \mathrm{y}$, en particular, sobre diferenciar cambios de ingreso positivos o negativos.

Para medir los efectos de choques de ingreso sobre el consumo se supone una especificación del proceso estocástico del ingreso en $\sum_{k=1}^{K} \varphi^{k} \pi_{i t}^{k}$, donde los parámetros $\varphi^{k}$ miden la magnitud del choque de ingreso y también se considera información sobre las estructuras del mercado del crédito y de activos o acciones. Las pruebas para la sensibilidad excesiva se han enfocado en discutir las condiciones para rechazar $\alpha=0$, que entre otras, pueden ser por factores estructurales o variables instrumentales que expliquen el ingreso y al mismo tiempo el consumo, y por omitir variables $z^{\prime}{ }_{i t}$ que sesguen la varianza condicional del crecimiento del consumo.

Aunque, la sensibilidad excesiva puede aparecer por la presencia de las imperfecciones del mercado del crédito, por medio de diferentes tasas de interés y por restricciones del crédito (Hall, 1978; Campbell \& Mankiw, 1989), con este enfoque, la mayoría de los estudios empíricos analizan la hipótesis con base en la identificación de choques permanentes y transitorios que se estiman con algún método de descomposición del ingreso, y aplicados a microdatos. En Krueger y Perri (2011), se plantea la pregunta de si el consumo de los hogares responde a choques de ingreso, por lo que construyen una medida de choques de ingreso laboral de corto y largo plazo para Italia, con una encuesta de hogares en panel de 1987 a 2008. Con la aplicación de modelos con funciones de distribución acumulada, encuentran que, los choques de ingreso laboral tienen modesta persistencia sobre el consumo, y pueden resolver sus problemas de financiamiento con créditos simples y con ahorros no contingentes. Para Ludwig (2015), la reacción del consumo de los hogares a choques transitorios y permanentes del ingreso disponible, depende del tipo del perfil de ingreso de los hogares. Con datos panel para el periodo 19982012 de Michigan, ellos encuentran que la respuesta a los choques de ingreso transitorio y permanente son muy diferentes entre hogares pobres y ricos, y concluyen que los hogares pobres en general reaccionan de manera similar a los dos tipos de choques de ingreso. Mientras que Commault (2017), analiza cómo afectan los choques transitorios de ingreso al consumo de los hogares, con una estrategia metodológica para reconciliar los resultados de métodos de experimentos naturales y estimaciones estructurales. Los métodos experimentales mostraron que el consumo responde a variaciones transitorias en el ingreso, tales como reembolso de impuestos o ganancia de lotería; y los métodos estructurales utilizan restricciones teóricas para identificar, que la elasticidad a choques transitorios del consumo aplicado a una muestra de datos es pequeña y no significativa.

Para el caso de la economía mexicana, existe un grupo de investigaciones que han estudiado los determinantes del consumo privado sin ninguna discusión sobre la sensibilidad excesiva al ingreso o efectos asimétricos (Galindo, 1993; 
Castro, et al., 1997; Ruiz-Galindo y Venegas-Martínez, 2007). Otras investigaciones destacan por el análisis de la durabilidad de los bienes del consumo, como el estudio de Campos y Meléndez (2013) que se enfoca en un análisis del consumo, de acuerdo con la hipótesis del ciclo de vida con funciones semiparamétricas aplicadas a bienes duraderos y no duraderos, $\mathrm{y}$ concluye que el consumo se comporta como U invertida que no es explicada por los cambios en la composición del hogar ni por la oferta laboral, y los resultados también sugieren que las restricciones de crédito son importantes para los hogares. En el estudio de Carbajal De Nova y Goicoechea (2014), se estiman elasticidades ingreso y del tipo de cambio real en bienes por durabilidad, nacionales e importados, y demuestra que elasticidades ingreso mayores a la unidad, dependen de la durabilidad de los bienes del consumo.

\section{Metodología para analizar la sensibilidad y asimetrías ante los choques de ingreso}

\subsection{La sensibilidad de largo plazo del consumo a cambios del ingreso corriente}

Con la metodológica que proponemos se identifican las dinámicas de corto plazo y el equilibrio de largo plazo de la sensibilidad del consumo a cambios del ingreso corriente. La hipótesis del ingreso absoluto se utiliza para definir que el consumo se determina por el ingreso corriente y los argumentos poskeynesianos, para incluir en la discusión la importancia del crédito al consumo vinculado, a la hipótesis de las restricciones de liquidez y liberación financiera y el tipo de cambio real, para el efecto de los precios relativos entre bienes nacionales e importados. Para identificar relaciones de largo plazo, suponemos que el consumo, el ingreso corriente, los créditos del consumo y los precios relativos cumplen con las condiciones de cointegración definidas por un vector que se escribe como la ecuación 1; y que una versión restringida con solo el ingreso corriente como variable explicativa también cumple con estar cointegrado. La función del consumo se especifica en doble logaritmo, por lo que se obtienen directamente las elasticidades ingreso corriente, crédito del consumo y tipo de cambio real; esto implica que la relación entre consumo e ingreso es no-lineal. Las elasticidades ingreso de las dos especificaciones se identifican como $\alpha_{1}^{* *}$, para la elasticidad ingreso en la función general y con $\alpha_{1}^{*}$, la elasticidad ingreso en la versión restringida con el ingreso corriente como única variable explicativa.

$$
c p_{t}=\alpha_{0}+\alpha_{1}^{* *} y_{t}+\alpha_{2} c r e_{t}-\alpha_{3} t c r_{t}+u_{t}
$$

En términos generales, si se cumple que $u_{t} \sim I(0)$, entonces el consumo y sus determinantes están cointegrados $\mathrm{y}$, por tanto, los parámetros alfa definen las elasticidades en el equilibrio de largo plazo. La hipótesis de ingreso absoluto 
se comprueba con la versión restringida, donde la elasticidad ingreso corriente define el equilibrio de largo plazo del consumo, cuando se cumple que sea significativa y mayor que cero $\left(\alpha_{1}^{*} \neq 0 ; \alpha_{1}^{*}>0\right)$; entonces, no son relevantes el crédito del consumo y el tipo de cambio real en la explicación del consumo en el largo plazo $\left(\alpha_{2}=\alpha_{3}=0\right)$. Mientras que la hipótesis de ingreso absoluto, en el marco poskeynesiano, implica que el ingreso corriente es importante $\left(\alpha_{1}^{* *} \neq 0 ; \alpha_{1}^{* *}>0\right)$ junto con los créditos del consumo y el tipo de cambio real en la determinación del consumo $\left(\alpha_{2} \neq 0 ; \alpha_{3} \neq 0\right)$.

Como referencia y para fines comparativos es importante mostrar que, en los estudios empíricos sobre la determinación del consumo por la teoría del ingreso permanente (Friedman, 1957), se propone una descomposición del ingreso corriente en dos partes no observadas, $y_{t}=y_{\text {per }, t}\left(\tau_{t}\right)+y_{\text {tra,t }}\left(c_{t}\right)$, donde $y_{\text {per }, t}$ es el ingreso permanente y $y_{\text {tra,t }}$, el ingreso transitorio. Porque si en la ecuación 1 se sustituye el ingreso corriente por el permanente, se obtienen las respectivas elasticidades ingreso en el largo plazo. Una de las estrategias que siguen estos estudios, para identificar los dos componentes del ingreso, es suponer que el ingreso permanente sigue un camino aleatorio con pendiente $\tau_{t}=\mu+\tau_{t-1}+v_{t}$ y el ingreso transitorio, un proceso autorregresivo $c_{t}=$ $\phi c_{t-1}+\varepsilon_{t}$, donde $v_{t}$ representa un choque aleatorio permanente y $\varepsilon_{t}$ un choque aleatorio transitorio (Urquiza, 2010; Krueger y Perri, 2011; Commault, 2017).

En contraposición al enfoque de la teoría del ingreso permanente, la existencia de relaciones de cointegración con fundamentos poskeynesianos que se especifican en la ecuación 1 y su versión restringida, implica que el consumo y el ingreso corriente, los créditos y el tipo de cambio real, se descompongan en comportamientos de largo y de corto plazo. La parte de largo plazo se define como una tendencia estocástica que tiene que ser común entre las variables del vector de cointegración, para que exista una combinación lineal estacionaria y una relación económica de largo plazo (Kirchgässner y Wolters, 2007). Lo interesante de esta lectura es que con un enfoque poskeynesiano, las elasticidades ingreso corriente de cointegración determinan el equilibrio de largo plazo y miden la sensibilidad del consumo a los cambios del ingreso corriente, de acuerdo con su tendencia estocástica común o comportamiento de largo plazo $\left(\tau_{t}\right)$.

De acuerdo con el planteamiento anterior, establecemos las siguientes predicciones sobre las elasticidades de largo plazo: 1). La elasticidad ingreso corriente de largo plazo del consumo pueden ser menor, igual o mayor a la unidad, dependiendo de si la propensión marginal de largo plazo mantiene la misma relación (menor, igual o mayor) con la propensión media de largo plazo; 2). La sensibilidad del consumo a cambios del ingreso corriente de largo plazo, $\left(\alpha_{1}^{*}>1 ; \alpha_{1}^{* *}>1\right)$, se acompaña por una propensión marginal mayor a la 
propensión media de largo plazo; 3). Definimos como la sobre-sensibilidad del consumo ante cambios del ingreso, en contra del concepto de sensibilidad excesiva ante cambios en el ingreso, si la elasticidad ingreso corriente es mayor a uno y las propensiones son iguales o mayores a 0.8 en el largo plazo; 4). Una de las razones de porque la elasticidad ingreso corriente de largo plazo es sobre-sensible, es cuando se incluye el ingreso y no se consideran otros factores explicativos. Por lo que al comparar las elasticidades ingreso de la ecuación 1 y su versión restringida, esperamos que se cumpla la regularidad en el largo plazo $\left(\alpha_{1}^{*}>\alpha_{1}^{* *}\right)$. Esta regularidad se muestra en la figura 1, donde la línea de relación de largo plazo del consumo, definida por la ecuación restringida, ahora se traslada hacia abajo por una elasticidad ingreso menor por la influencia del crédito del consumo, y el tipo de cambio real, y con el supuesto de la misma ordenada al origen $\left.\left(\alpha_{0}\right) ; 5\right)$. Para que las predicciones poskeynesianas del consumo se cumplan, se tiene que confirmar que la elasticidad ingreso corriente sea mayor a las elasticidades de crédito del consumo y tipo de cambio real en el largo plazo $\left|\alpha_{1}^{* *}\right|>\left|\alpha_{2}\right| ;\left|\alpha_{3}\right|$.

Una vez indicado el equilibrio de largo plazo del consumo y el ingreso corriente, lo que se encuentre fuera de equilibrio se identifica como una relación de corto plazo y con componentes cíclicos. La línea gruesa en la figura 1 contiene las combinaciones de niveles del consumo e ingreso de equilibrio, con las elasticidades de largo plazo de la ecuación 1. En tal marco analítico, todos los puntos por arriba y por debajo de la línea gruesa representan niveles de consumo, dado el ingreso corriente que se encuentran fuera del equilibrio de largo plazo y que definimos como relaciones económicas de corto plazo. Los niveles del consumo en desequilibrio pueden deberse a diferentes situaciones, las que donde se muestran en dos tipos de modificaciones o cambios del ingreso corriente: temporal y continuo, que analíticamente sustituyen a los conceptos de transitorio y permanente.

Para analizar la dinámica de los desequilibrios del consumo de corto plazo se utilizan dos puntos de equilibrios de referencias, identificados como $\mathrm{E}_{1}$ y $\mathrm{E}_{2}$; $\mathrm{y}$, cuatro puntos de desequilibrios con dos situaciones del consumo por arriba del equilibrio $\left(\mathrm{A}_{1}\right.$ y $\left.\mathrm{A}_{2}\right)$ y otros dos por debajo del equilibrio de largo plazo $\left(\mathrm{B}_{1}\right.$ y $\mathrm{B}_{2}$ ). El primer caso representado por el punto $\mathrm{A}_{1}$ indica que con el mismo nivel de ingreso corriente se puede obtener un nivel del consumo de equilibrio $E_{1}$, pero también indica la posibilidad de un nivel del consumo por arriba de equilibrio, que puede ser financiado con un crédito y el patrón del consumo después regresa al nivel de ingreso corriente. En el siguiente escenario, se supone que el ingreso corriente puede aumentar de forma temporal o continua de $E_{1}$ a $E_{2}$; esto es, el ingreso corriente puede aumentar por un cambio en el ingreso salarial por un bono de un mes y regresa al nivel de ingreso salarial anterior, o por un bono que se mantiene de manera continua para todos los meses. En el primer caso, el incremento temporal del ingreso corriente 
provoca que el consumo correspondiente aumente hasta el punto $\mathrm{A}_{2} \mathrm{y}$ tienda a reducirse hasta encontrar nuevamente el nivel del consumo de largo plazo en $\mathrm{E}_{1}$; mientras con el nuevo nivel de ingreso laboral, el consumo en $\mathrm{E}_{1}$ puede aumentar hasta $\mathrm{E}_{2}$ sobre la línea de equilibrio de largo plazo.

En el mismo esquema, donde el incremento del ingreso corriente es temporal, pero se transforma en un ingreso con dinámica continua, entonces se observa una sobre-reacción del consumo identificado en $\mathrm{A}_{2}$ y posterior una tendencia hacia el nuevo equilibrio del consumo de largo plazo identificado por E2. Los puntos $\left(\mathrm{B}_{1}\right.$ y $\left.\mathrm{B}_{2}\right)$ nos permiten analizar los efectos de una reducción del ingreso sobre el consumo en el corto y largo plazo. En el caso de una reducción temporal del ingreso corriente $\mathrm{E}_{2}$ a $\mathrm{E}_{1}$, el nivel del consumo se puede contraer hasta el punto de equilibrio $\mathrm{E}_{1}$, o puede disminuir hasta el punto $\mathrm{B}_{1}$ de sobrereacción del consumo, en el corto plazo, y recorrer distancias diferentes camino al nivel del consumo de equilibrio inicial en $\mathrm{E}_{2}$. Pero si la reducción del ingreso corriente tiene un comportamiento continuo, el punto $\mathrm{B}_{1}$ de sobre-reacción del consumo tiene una ruta de regreso al equilibrio mayor que la que sigue el consumo en $\mathrm{E}_{2}$ hacia el nuevo equilibrio de largo plazo en $\mathrm{E}_{1}$. El consumo de corto plazo en el punto $\mathrm{B}_{2}$ indica una disminución temporal del ingreso, que reduce el nivel de consumo por debajo del equilibrio y regreso al equilibrio inicial en E2. Pero si la reducción temporal se convierte en un proceso continuo, entonces el consumo se dirige al nuevo equilibrio $\mathrm{E}_{1}$, y la distancia es mayor que si sigue la ruta directa del punto de equilibrio $\mathrm{E}_{2}$ hacia $\mathrm{E}_{1}$.

\subsection{La sensibilidad de corto plazo del consumo ante cambios del ingreso corriente}

El análisis de las trayectorias del consumo de corto plazo hacia el consumo de equilibrio de largo es relevante para entender la vinculación entre las sensibilidades ingreso del consumo de corto plazo y largo plazo, y sobre todo para formalizar las relaciones económicas de corto y largo plazo, representadas por las elasticidades ingreso corriente. Como se estableció, las elasticidades ingreso de equilibrio de largo plazo $\left(\alpha_{1}^{* *}, \alpha_{1}^{*}\right)$ se obtienen de la relación de cointegración general y restringida de la ecuación 1, y se identifican por las líneas continuas y punteadas en negro de la figura 1. Una estrategia general para analizar las elasticidades de corto plazo es suponer que las distancias de todos los niveles del consumo en desequilibrio tipo A y B son equivalentes o simétricos, lo cual implica que es suficiente suponer que existe una elasticidad ingreso de corto plazo, con tendencia hacia la elasticidad de equilibrio de largo plazo, cuando se cumplen las condiciones de convergencia. 


\section{Figura 1}

\section{Desequilibrios de corto plazo y relación consumo e ingreso de largo plazo}

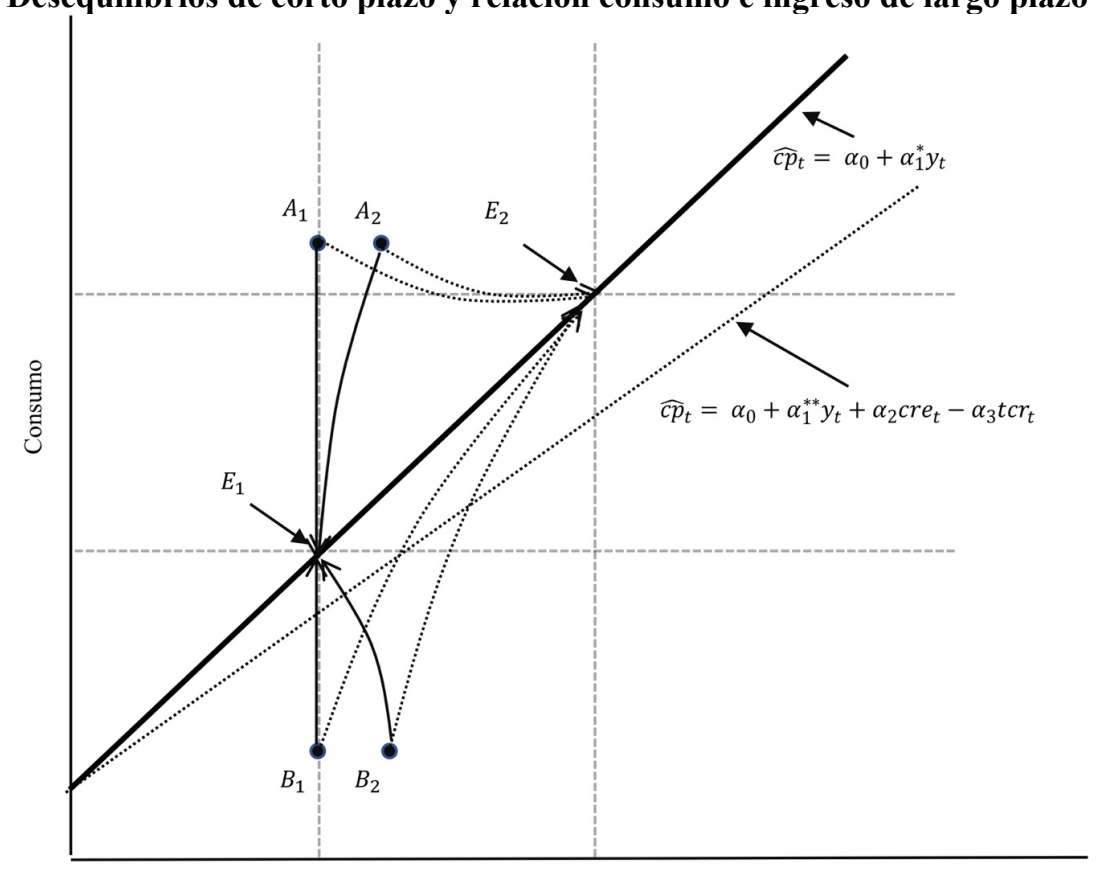

Ingreso corriente

Fuente: Elaboración propia

En la ecuación 2, se presentan la especificación del modelo de corrección de error (mce), con la primera diferencia $(\Delta)$ del consumo y sus determinantes, lo cual supone que en todos los casos son $\mathrm{I}(0)$; con parámetros como elasticidades ingreso corriente de corto plazo $\left(\beta_{1}^{* *}, \beta_{1}^{*}\right)$, que están condicionadas a las elasticidades de largo plazo $\left(\alpha_{1}^{* *}, \alpha_{1}^{*}\right)$, definidas por la ecuación de cointegración 1 y su versión restringida. Por el teorema de representación de Engle-Granger, las elasticidades ingreso corriente de corto plazo tienden a las de largo plazo, siempre y cuando el parámetro $\gamma$ sea significativo, negativo y en términos absolutos entre cero y uno. Dado que las variables en primera diferencia se definen en $t$, se garantiza que la elasticidad de corto plazo tiene tendencia asintótica hacia la de largo plazo.

$$
\begin{gathered}
\Delta c p_{t}=\beta_{0}+\beta_{1}^{* *} \Delta y_{t}+\beta_{2} \Delta c r e_{t}-\beta_{3} \Delta t c r_{t} \\
-\gamma\left(c p_{t-1}-\alpha_{0}-\alpha_{1}^{* *} y_{t-1}-\alpha_{2} c r e_{t-1}+\alpha_{3} t c r_{t-1}\right)+\varepsilon_{t}
\end{gathered}
$$


Figura 2

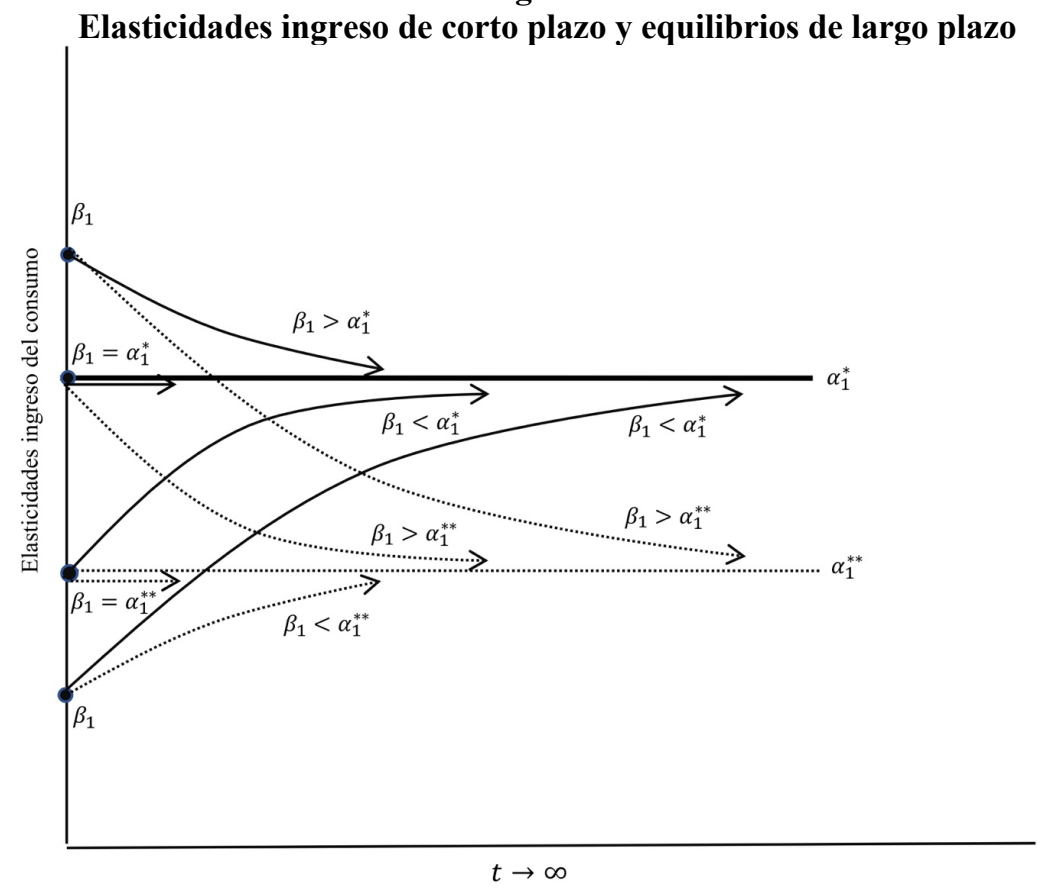

Fuente: Elaboración propia

Las elasticidades ingreso de corto plazo cumplen las mismas condiciones que las de largo plazo, en términos de sobre-sensibilidad $\left(\beta_{1}^{*}>1 ; \beta_{1}^{* *}>1\right)$; pero, en el comportamiento dinámico, es importante suponer escenarios con elasticidad ingreso de corto plazo igual, mayor o menor a la de largo plazo. En la figura 2, se definen las dos elasticidades de largo plazo de la ecuación 1 y su versión restringida, donde se mantiene el supuesto $\alpha_{1}^{*}>\alpha_{1}^{* *}$, por el efecto de incluir las variables del crédito del consumo y el tipo de cambio real. En el escenario donde la elasticidad de corto plazo es igual a la de largo plazo $\left(\beta_{1}=\right.$ $\left.\alpha_{1}^{*} ; \beta_{1}=\alpha_{1}^{* *}\right)$ no existe una dinámica, debido a que se observan las condiciones de equilibrio donde los consumidores absorben el efecto total de un cambio en el ingreso corriente. Pero si con el cambio del ingreso se modifica la elasticidad ingreso de largo plazo, en cualquier dirección $\left(\alpha_{1}^{*} \leftrightarrow \alpha_{1}^{* *}\right)$, entonces la elasticidad de corto plazo tiende al nuevo equilibrio de largo plazo y es simétrico en términos de distancia y tiempo (fig. 2). Cuando la elasticidad de corto plazo es mayor (menor) a las dos elasticidades de largo plazo $\left(\beta_{1}>\alpha_{1}^{*}>\alpha_{1}^{* *} ; \beta_{1}<\alpha_{1}^{*}<\alpha_{1}^{* *}\right)$, lo que se observa es un recorrido con menor distancia y tiempo al equilibrio más cercano, y lo contrario sucede al equilibrio más lejano; $\mathrm{y}$, como se observa en la figura 2 , son comportamientos 
simétricos. En todos los casos, lo que restringe la velocidad de convergencia es el parámetro de ajuste al equilibrio $\gamma$.

\subsection{Sensibilidades asimétricas de corto plazo a cambios del ingreso corriente}

La elasticidad ingreso de corto plazo depende del parámetro de ajuste al equilibrio y de la elasticidad de largo plazo que se obtienen de estimar el modelo de corrección de error, con rezagos de las primeras diferencias del consumo y las variables exógenas. Pero, cuando se consideran más de un parámetro de ajuste al equilibrio $\left(\gamma_{L}, \gamma_{M}, \gamma_{H}\right)$, en los modelos de corrección de error, entonces se detectan desequilibrios asimétricos y diferentes elasticidades ingreso de corto plazo, las cuales representan las posiciones por arriba o por debajo con respecto al equilibrio; como son los puntos A y B, respectivamente, en la figura 1. Es importante mencionar que la ventaja de especificar la ecuación 3 con rezagos para todas las variables de $t-1$ hasta $t-p$, es la consistencia con la metodología de cointegración de Johansen (1992), donde se identifica a la ecuación 3 como la primera del sistema de ecuaciones del Vector de Corrección de Error (VEC por sus siglas en inglés). En un enfoque analítico, donde el ingreso corriente inicie en $t-1\left(\Delta y_{t-1}\right)$, en lugar de $t$ como en la ecuación 2, tiene como consecuencia que la elasticidad de corto plazo se entienda como el efecto con un rezago de un cambio del ingreso corriente sobre el consumo, y que su medición no sea el parámetro $\beta_{1}^{* *}$, sino ahora involucra el parámetro de ajuste al equilibrio y la elasticidad ingreso de largo plazo $\left(\gamma \alpha_{1}^{* *}+\beta_{1}^{* *}\right)$. Para que exista sobre-sensibilidad del consumo $(>1)$ a modificaciones del ingreso corriente en el corto plazo, deben suceder las siguientes combinaciones en los parámetros que definen la elasticidad de corto plazo; 1) los tres parámetros estimados tengan valores numéricos iguales o mayor de $0.7 ; 2$ ) si los parámetros de ajuste al equilibrio y la elasticidad ingreso de largo plazo crecen por arriba de 0.7 , entonces el parámetro $\beta_{1}^{* *}$ puede estar en el rango $0.2-0.5 ; \mathrm{y}, 3)$ con valores mayores a 0.8 en el parámetro $\beta_{1}^{* *}$, los parámetros de ajuste al equilibrio y la elasticidad ingreso de largo plazo, en conjunto, pueden ser menores a 0.5 .

El análisis anterior sobre la elasticidad ingreso de corto plazo supone que existe una elasticidad ingreso de largo plazo, -se tiene la posibilidad de una elasticidad ingreso de largo plazo sin crédito al consumidor y tipo de cambio real $\alpha_{1}^{*}$ - un $\beta_{1}$ y en especial el parámetro de ajuste de equilibrio $\gamma$ que determina si la dinámica es homogénea o simétrica al equilibrio. De acuerdo con la metodología de regímenes múltiples de cointegración (threshold cointegration), con modelos con vectores de corrección de error (thresholds$V E C M$ ) propuestos por Hansen y Seo (2002) y Seo (2011), establecemos dos especificaciones de la función del consumo, para identificar elasticidades ingreso de corto plazo, con dinámica asimétrica hacia el equilibrio de largo 
plazo. En la primer especificación de la función del consumo, se considera la posibilidad de dos regímenes; el primero es para identificar los desequilibrios en los puntos A de la figura 1, que podrían estar relacionadas en términos del ciclo ascendente con consumo e ingreso en auge económico; y, el régimen segundo para los desequilibrios tipo punto $\mathrm{B}$ de la figura 1, para momentos de desaceleración y/o crisis económica. En la ecuación 3, se definen los regímenes con dos parámetros de ajuste al equilibrio; con el parámetro de ajuste $\gamma_{L}$ se identifica la situación de desequilibrios, por debajo $(L)$ y $\gamma_{H}$ para los desequilibrios por arriba $(H)$ de la relación de largo plazo. En esta especificación, se supone que la relación de cointegración es la misma, los parámetros de las variables endógenas y exógenas rezagadas se estiman con mismo VEC, por lo que estos son iguales para los dos regímenes. En el caso de parámetros de ajuste a los equilibrios diferentes $\left(\gamma_{L} \neq \gamma_{H}\right)$, la velocidad es más lenta al equilibrio en el régimen con el parámetro de ajuste mayor, por lo que la consecuencia será una elasticidad ingreso de corto plazo mayor. Este resultado implica suponer que el consumo se ajusta más lento al equilibrio de largo plazo $\left(\gamma_{L}>\gamma_{H}\right)$, para que la elasticidad ingreso de corto plazo sea mayor en un régimen de crisis económica $\left(\gamma_{L} \alpha_{1}^{* *}+\beta_{1}^{* *}\right)>\left(\gamma_{H} \alpha_{1}^{* *}+\beta_{1}^{* *}\right)$.

$$
\begin{gathered}
\Delta c p_{t}=\beta_{0}+\sum_{i=1}^{p} \phi_{i} \Delta c p_{t-i}+\sum_{i=1}^{p} \beta_{1, i}^{* *} \Delta y_{t-i}+\sum_{i=1}^{p} \beta_{2, i} \Delta c r e_{t-i}- \\
\sum_{i=1}^{p} \beta_{3, i} \Delta t c r_{t-i}-\left\{\begin{array}{l}
\gamma_{L} V C_{L, t-1}+\varepsilon_{t}, \\
\gamma_{H} V C_{H, t-1}
\end{array}\right.
\end{gathered}
$$

donde el vector de cointegración definido por la ecuación 1 se escribe como

$$
V C_{t-1}=\left(c p_{t-1}-\alpha_{0}-\alpha_{1}^{* *} y_{t-1}-\alpha_{2} c r e_{t-1}+\alpha_{3} t c r_{t-1}\right) .
$$

Para analizar el caso de desequilibrios muy cercanos (por arriba y debajo) de la relación de largo plazo, se añade un nuevo régimen $\gamma_{M}$ a los dos tipos anteriores. La metodología que definimos, de acuerdo con Hansen y Seo (2002) y Seo (2011), es la de mantener el supuesto de la relación de cointegración y estimar los parámetros de las variables endógenas y exógenas rezagadas con diferentes modelos VEC (VECM). Como se muestra en la ecuación 4, la especificación supone la misma elasticidad ingreso de largo plazo y dos fuentes de variación entre regímenes, los parámetros de la correlación del consumo y el ingreso con rezago y los de ajuste al equilibrio $\left(\beta_{L, 1}^{* *}, \beta_{M, 1}^{* *}, \beta_{H, 1}^{* *}\right)$ y $\left(\gamma_{L}, \gamma_{M}, \gamma_{H}\right)$-, para estimar las elasticidades de corto plazo. En un escenario de crisis económica donde los consumidores se ajustan lentamente al equilibrio de largo plazo y reaccionan fuertemente a cambios ingreso de corto plazo, entonces se espera una elasticidad ingreso de corto plazo mayor con la siguiente combinación de condiciones: 1) si los agentes ajustan más lento el consumo en el régimen bajo, se espera que se cumpla que $\gamma_{L}>\gamma_{M}>\gamma_{H}$;2) que la correlación entre el consumo y el ingreso rezagado sea mayor en el régimen bajo, lo cual implica que $\left.\beta_{L, 1}^{* *}>\beta_{M, 1}^{* *}>\beta_{H, 1}^{* *} ; \mathrm{y}, 3\right)$ la 
elasticidad ingreso de corto plazo, por tanto, será mayor en el régimen bajo $\left(\gamma_{L} \alpha_{1}^{* *}+\beta_{L, 1}^{* *}\right)>\left(\gamma_{M} \alpha_{1}^{* *}+\beta_{M, 1}^{* *}\right)>\left(\gamma_{H} \alpha_{1}^{* *}+\beta_{H, 1}^{* *}\right)$. Aunque el escenario anterior es el más claro, se pueden definir comportamientos distintos con otras combinaciones de parámetros y como resultado se encuentre una elasticidad ingreso de corto plazo mayor en el régimen bajo. Un caso, es suponer que los consumidores ajustan más rápido al equilibrio de largo, lo cual implica que las correlaciones entre el consumo y el ingreso rezagado serán suficientemente más altas, para que la elasticidad ingreso de corto plazo siga siendo mayor en el régimen bajo.

$$
\begin{aligned}
& \left\{\begin{array}{l}
\Delta c p_{L, t} \\
\Delta c p_{M, t} \\
\Delta c p_{H, t}
\end{array}=\left\{\begin{array}{l}
\beta_{L, 0} \\
\beta_{M, 0} \\
\beta_{H, 0}
\end{array}+\left\{\begin{array}{l}
\sum_{i=1}^{p} \phi_{L, i} \Delta c p_{t-i} \\
\sum_{i=1}^{p} \phi_{M, i} \Delta c p_{t-i} \\
\sum_{i=1}^{p} \phi_{H, i} \Delta c p_{t-i}
\end{array}+\left\{\begin{array}{l}
\sum_{i=1}^{p} \beta_{L, 1, i}^{* *} \Delta y_{t-i} \\
\sum_{i=1}^{p} \beta_{M, 1, i}^{* *} \Delta y_{t-i}+ \\
\sum_{i=1}^{p} \beta_{H, 1, i}^{* *} \Delta y_{t-i}
\end{array}\right.\right.\right.\right. \\
& \left\{\begin{array}{l}
\sum_{i=1}^{p} \beta_{L, 2, i} \Delta c r e_{t-i} \\
\sum_{i=1}^{p} \beta_{M, 2, i} \Delta c r e_{t-i} \\
\sum_{i=1}^{p} \beta_{H, 2, i} \Delta c r e_{t-i}
\end{array}-\left\{\begin{array}{l}
\sum_{i=1}^{p} \beta_{L, 3, i} \Delta t c r_{t-i} \\
\sum_{i=1}^{p} \beta_{M, 3, i} \Delta t c r_{t-i} \\
\sum_{i=1}^{p} \beta_{H, 3, i} \Delta t c r_{t-i}
\end{array}-\left\{\begin{array}{l}
\gamma_{L} V C_{L, t-1} \\
\gamma_{M} V C_{M, t-1} \\
\gamma_{H} V C_{H, t-1}
\end{array}+\left\{\begin{array}{l}
\varepsilon_{L, t} \\
\varepsilon_{M, t} \\
\varepsilon_{H, t}
\end{array}\right.\right.\right.\right.
\end{aligned}
$$

\subsection{Estadísticos de pruebas para cointegración threshold-VECM (TVECM)}

De acuerdo con la estimación de un modelo vector de corrección de error bivariado con regímenes (TVECM), se han diseñado estadísticos para contrastar dos pruebas de cointegración (cointegración-threshold): 1) cointegración lineal versus cointegración-threshold; y, 2) no cointegración versus cointegración-threshold, las cuales se usarán para concluir si existe la evidencia de dos parámetros de ajuste al equilibrio, relativamente distintos, $\left(\gamma_{L}, \gamma_{H}\right)$ y comportamientos asimétricos de las elasticidades de corto plazo hacia el largo plazo. Para el primer caso, Hansen y Seo ( 2002) propusieron utilizar una prueba sup-LM para comparar un VECM lineal versus threshold$V E C M$ con dos regímenes, donde Stigler (2013) indica que el vector de cointegración es desconocido; pero, es suficiente utilizar un TVECM bivariante para obtener el valor máximo adecuado del sup-LM. Aunque la prueba involucra modelos de corrección de error para el consumo y el ingreso corriente, utilizamos el estadístico sup-LM para probar la hipótesis nula $\gamma_{L}=$ $\gamma_{H}$ entonces, se acepta una relación de cointegración lineal con ajuste al equilibrio simétrico, en contraste con la alternativa de la relación de cointegración con ajuste al equilibrio de largo plazo asimétrico $\left(\gamma_{L} \neq \gamma_{H}\right)$. El planteamiento de la hipótesis nula y alternativa en Seo (2006) es importante analizarlo en el contexto de análisis de cointegración, debido a que el resultado de probar la hipótesis de no cointegración versus cointegración-threshold, podría llevar a la inferencia general de no encontrar cointegración. El estadístico sup-Wald se propone para probar la hipótesis nula $\gamma_{L}=\gamma_{H}=0$ con respecto a la alternativa $\gamma_{L}=\gamma_{H} \neq 0$, considerando el vector de cointegración. 


\subsection{Datos y análisis exploratorio del consumo privado e ingreso}

Los datos para las variables del consumo, el ingreso corriente, los créditos del consumo y el tipo de cambio real provienen del INEGI y Banxico. El consumo se mide con los índices del volumen físico mensual del consumo privado, bienes duraderos, semi-duraderos, no-duraderos y servicios de origen nacional, y bienes duraderos, semi-duraderos y no duraderos de origen importados con año base 2013=100, para el periodo enero de 1995 a septiembre de 2017. Como un buen indicador del pib (Elizondo, 2019) y del ingreso corriente, se utiliza el indicador global de actividades económicas (igae) con año base 2013 $=100^{1}$; el crédito del consumo ( $\mathrm{cre}$ ) es la suma de los créditos de la banca comercial otorgados con tarjetas de crédito, para bienes del consumo duradero y otros, que originalmente se obtuvieron en saldos a millones de pesos, se transformaron a precios de 2013 con el inpc y en índices base $2013=100$. Por último, el índice de tipo de cambio real se mide con el tipo de cambio nominal pesos por dólar, ponderado con una canasta de índices de precios al consumidor con respecto a 111 países (Banxico).

Para analizar las tendencias de las propensiones media y marginal en el periodo de análisis, se calculó primero la propensión media como el consumo privado entre el PIB a precios constantes de 2013; en segundo lugar, se suponen tres tipos de elasticidades ingreso para analizar el caso de sensibilidad baja (0.85), unitaria (1.0) y de sobre-sensibilidad (1.28) del consumo privado a modificaciones del ingreso corriente; $\mathrm{y}$, por último, se estiman las propensiones marginales correspondientes. El escenario básico es cuando la elasticidad ingreso es la unidad y, por tanto, la propensión marginal es igual a la propensión media. El crecimiento acelerado de la propensión media -a partir de 1995- muestra claramente el cumplimiento sobre la predicción de mayor consumismo por la apertura comercial (Arrau \& Oks, 1992); se alcanza el máximo en el periodo previo a la crisis de 2009 y, posterior a ella, se observa una tendencia decreciente. En este escenario, no se observa una situación de sensibilidad del consumo privado a cambios del ingreso, combinada con propensiones media y marginal relativamente altas, como las que se muestran en la figura 3, que se mantienen en el rango de 0.6 a 0.7 . Es claro que en el escenario alternativo, donde se supone una elasticidad ingreso menor a la unidad (0.85), el riesgo de una propensión marginal relativamente alta se descarta. Sin embargo, el tercer escenario con una elasticidad ingreso mayor a la unidad (1.28), muestra claramente cómo la propensión marginal a consumir, se encuentra en una zona de "riesgo" entre 0.8 y 0.9 , lo cual indicaría sobresensibilidad de los consumidores a choques de ingreso de corto plazo, en el periodo de apertura comercial.

${ }^{1}$ En la mayoría de los estudios, sobre el consumo en México, existe el consenso de utilizar al Producto Interno Bruto (PIB) como un buen indicador de ingreso. Sin embargo, Elizondo (2012 y 2019) demuestra que el IGAE es la mejor manera para predecir el PIB mensual. 
Con el análisis exploratorio de regresiones con doble logaritmo y diagramas de dispersión (fig. 4), resalta la posibilidad de un escenario de sobresensibilidad de la función consumo privado, por la presencia de una elasticidad ingreso mayor a la unidad (1.15) que implica una propensión marginal a consumir, mayor a la propensión media y una nueva frontera máxima de 0.8 en el periodo 2002-2008, con un patrón de riesgo del consumo con alta sensibilidad a choques de ingreso (Campbell \& Mankiw, 1989), previo a la crisis de 2009 en la economía mexicana. Este resultado contrasta con la elasticidad ingreso unitaria encontrada por otros estudios (Galindo, 1993; Castro, et al., 1997; Ruiz-Galindo y Venegas-Martínez, 2007), con datos para periodos distintos, previos a las crisis de 1995 y de ajuste económico de 2001 y donde, en muchos casos, el consumo privado depende solo del ingreso corriente.

\section{Figura 3}

Tendencias de las propensiones media y marginal en México, 1995-2017

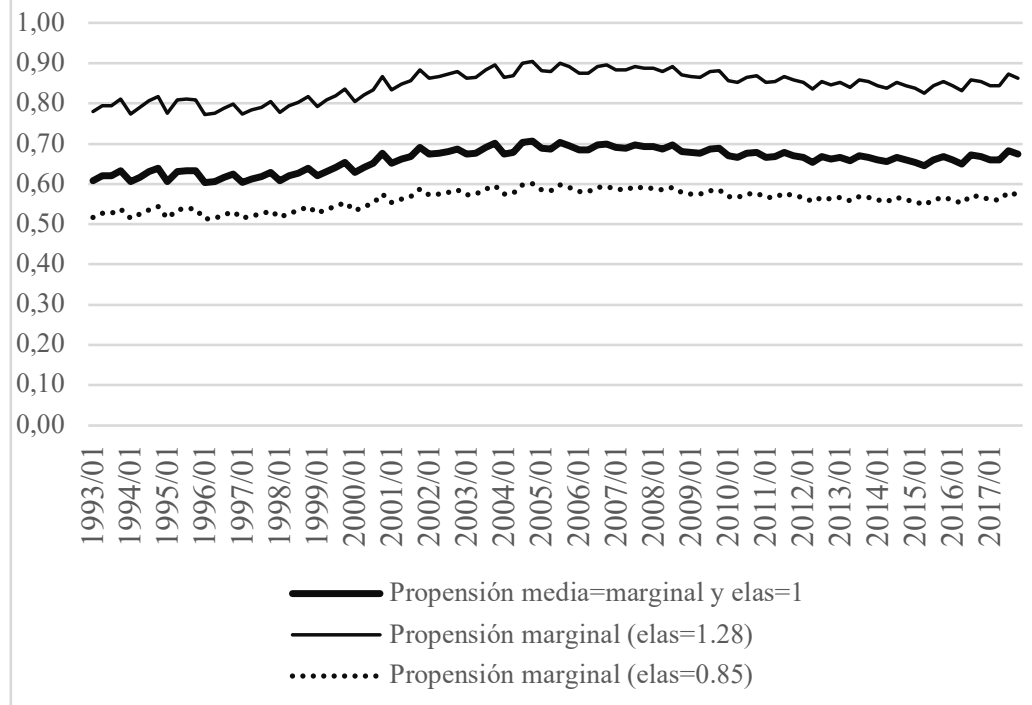

Fuente: Elaborado con base a datos del INEGI

En el caso del consumo de bienes desagregado por durabilidad y de procedencia interno o importado, algunas de las predicciones indican que las regularidades esperadas son bienes del consumo de mayor durabilidad con tendencia a mayor sensibilidad a los choques de ingreso y a que los bienes del consumo de producción interna mantengan una elasticidad ingreso menor, en comparación con los importados; sobre todo, en el marco de apertura comercial (Mallick y Mohsin, 2016; Carbajal De Nova y Goicoechea, 2014). El diagrama 
y la regresión estimada muestran que la elasticidad ingreso del consumo de servicios, es muy parecida a la del consumo privado (figura 4). Pero cuando se analizan los bienes del consumo por su durabilidad, los resultados muestran elasticidades ingreso más altas en los bienes durables producidos internamente o importados. En particular, los bienes semi-duraderos nacionales tienden a no cumplir con tal predicción, dado que su elasticidad ingreso es menor a los bienes no-duraderos; resalta la mayor dispersión alrededor de la línea de regresión que se muestra en el diagrama de dispersión correspondiente, en la figura 4. La segunda predicción también se cumple al comparar que las elasticidades ingreso de los bienes del consumo de producción interna están en el rango de 0.7 a 1.5 por durabilidad, mientras que las elasticidades ingreso de los bienes importados se estimaron entre 2.7 y 4.5, también ordenadas por su durabilidad. Es importante mencionar que esperamos confirmar estas predicciones con los resultados de la aplicación de la metodología, y ponderarlas cuando se incluyan los factores explicativos de los créditos del consumo y el tipo de cambio real. En el primer caso, debido a que la liberación financiera provocó el aumento del crédito del consumo a partir de la década del 2000 en México (Miller, 2013; de la Cruz y Alcántara, 2011) y, como consecuencia, se aceleró la compra de bienes no-duraderos y duraderos, nacional e importados. Mientras que la apertura comercial influyó para que la demanda de bienes importados y de producción interna, que dependen de insumos y bienes de capital importados, se volvieran más sensibles al tipo de cambio (Mendoza-González, 2020; Castro, et al., 1997).

\section{Resultados}

En esta parte, se presentan los resultados de la aplicación de la metodología econométrica en tres fases: en la primera, se aplica el análisis de integración para identificar el orden de integración de todas las variables exógenas del consumo privado y de las variables explicativas; en la segunda fase, se utiliza la metodología de (Johansen \& Juselius, 1990) para probar que los distintos bienes del consumo y las variables exógenas representan relaciones de largo plazo con solo un vector de cointegración, y se estiman funciones del consumo de largo plazo sin sesgo de endogeneidad (Spanos, 2003) de las variables ingreso corriente, crédito del consumo y tipo de cambio real con el método de estimación FMOLS (Phillips \& Hansen, 1990; Saikkonen, 1991); y, finalmente se analiza la dinámica de las elasticidades de corto plazo con regímenes múltiples con base en las pruebas bivariadas de Seo (2006), para la hipótesis nula de no cointegración y alternativa de cointegración-threshold, y la Hansen-Seo (2002) para la hipótesis nula cointegración lineal con respecto a cointegración-threshold, y se estiman modelos con múltiples regímenes tipo TVECM (Seo, 2011; Stigler, 2013). 


\section{Figura 4}

Diagramas de dispersión del consumo privado agregado, servicios y bienes por durabilidad con respecto al ingreso, 1995-2017

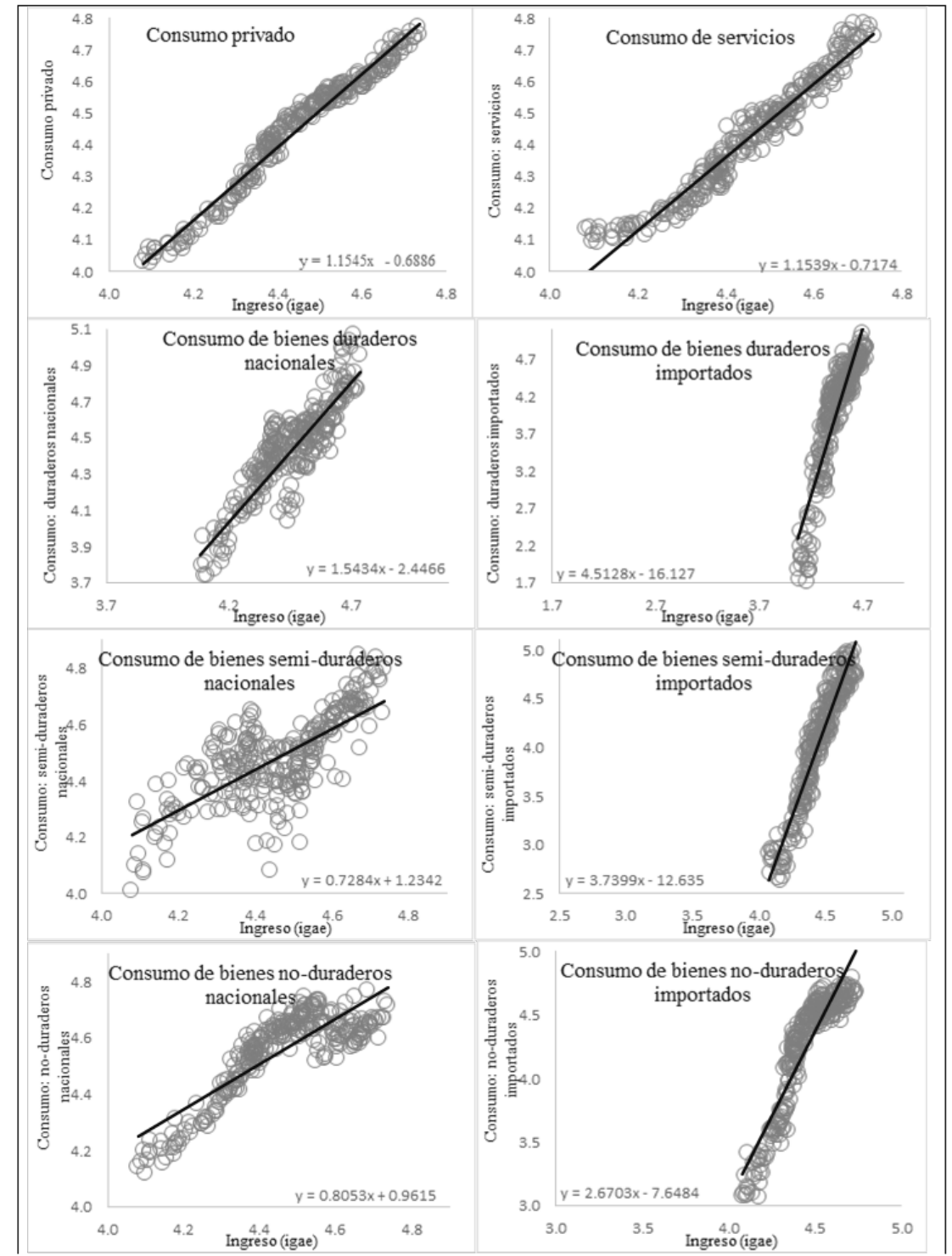

Fuente: Elaboración propia con base a datos del INEGI 


\subsection{Análisis de integración}

Para el análisis de integración se aplicaron las pruebas de raíz unitaria DickeyFuller aumentada -ADF- (Dickey y Fuller, 1979; Dickey y Fuller, 1981), Philips-Perron -PP- (Phillips y Perron, 1988), Elliott, Rothenberg y Stock ERS- (Elliott, et al., 1996) y para estacionariedad de Kwiatkowski, Phillips, Schmidt y Shin -KPSS- (Kwiatkowski, et al., 1992) a las variables en logaritmo para el consumo privado y su desagregación, y las variables ingreso corriente, crédito al consumidor y el tipo de cambio real. Los tres primeros estadísticos se utilizan para analizar la hipótesis nula de raíz unitaria y con el estadístico KPSS la hipótesis nula de estacionariedad. Los resultados de la aplicación de las pruebas que se presentan en el cuadro 1, muestran que el consumo privado, el consumo de servicios, los bienes duraderos, semi-duraderos y no-duraderos nacionales e importados, son estacionarios con la primera diferencia del logaritmo de la variable, por lo que tienen un orden de integración igual a uno, $c p_{t} \sim I(1)$. Para las variables del ingreso corriente (igae) y el tipo de cambio real (tcr), también se encontró un orden de integración de uno igae y tcr $\sim I(1)$, pero para el crédito del consumo se aplicó una segunda diferencia para que cumpliera con ser estacionaria; por tanto, su orden de integración fue de dos - cre $\sim I(2)$. En una investigación previa (MendozaGonzález, 2020) se muestra que el orden de integración superior encontrado, se relaciona con el ciclo en el crecimiento de los créditos del consumo previo a la crisis económica de 2009 y, desde el enfoque econométrico, se recomienda incluir la variable de crédito junto con la variable ingreso para asegurar un vector de cointegración.

\subsection{Análisis de cointegración de Johasen y Juselius con VECM}

La metodología de Johasen y Juselius - $J J$ - (Johansen \& Juselius, 1990) utiliza un $V E C M$ para estimar los parámetros de ajuste $(\gamma)$ por cada variable endógena que supone una dinámica de las relaciones de corto plazo, condicionado de forma simétrica al equilibrio de largo plazo. Con tal esquema analítico, se aplicó el procedimiento de lo general a lo particular en la metodología $J J$, para probar la sensibilidad de la relación de cointegración cuando se reduce el número de variables exógenas. En el nivel de análisis general, se aplicaron las pruebas de $J J$ para cada tipo del consumo y se incluyeron todas las variables exógenas: ingreso corriente (ligae), crédito del consumo (lcre) y el tipo de cambio real (ltcr); en el segundo nivel, se aplican las pruebas con la combinación de las variables exógenas, ingreso corriente y créditos y, por el otro lado, con el ingreso corriente y el tipo de cambio; por último, se aplican las pruebas a la relación entre el consumo y el ingreso corriente. 


\section{Cuadro 1}

Análisis de integración para consumo por durabilidad y origen, ingreso, crédito al consumo y tipo de cambio real

\begin{tabular}{|c|c|c|c|c|c|c|c|c|}
\hline Variables & ADF & V. crítico & $\mathrm{PP}$ & V. crítico & ERS & V. crítico & KPSS & V. críticc \\
\hline \multicolumn{9}{|l|}{ Consumo privado agregado } \\
\hline Total (cpt) & -2.57 & -3.42 & -5.95 & -3.42 & -1.38 & -2.89 & 0.41 & 0.15 \\
\hline 1er Dif. (cpt) & -4.24 & & -43.82 & & -3.49 & & 0.06 & \\
\hline Consumo de servicios (csn) & -2.15 & & -7.95 & & -2.99 & & 0.17 & \\
\hline 1er Dif. (csn) & -3.80 & & -28.16 & & -3.15 & & 0.05 & \\
\hline \multicolumn{9}{|l|}{ Bienes duraderos } \\
\hline Nacionales (cp_dur_bn) & -2.74 & & -6.03 & & -2.98 & & 0.23 & \\
\hline 1er Dif. (cp_dur_bn) & -4.92 & & -32.98 & & -5.46 & & 0.08 & \\
\hline Importados (cp_dur_bi) & -3.15 & & -3.76 & & -2.45 & & 0.38 & \\
\hline 1er Dif. (cp_dur_bi) & -4.99 & & -23.59 & & -6.03 & & 0.04 & \\
\hline \multicolumn{9}{|l|}{ Bienes semi-duraderos } \\
\hline Nacionales (cp_semidur_bn) & -1.85 & & -6.63 & & -2.48 & & 0.23 & \\
\hline 1er Dif. (cp_semidur_bn) & -3.70 & & -31.43 & & -9.23 & & 0.08 & \\
\hline Importados (cp_semidur_bi) & -2.00 & & -5.89 & & -3.39 & & 0.42 & \\
\hline 1er Dif. (cp_semidur_bi) & -5.36 & & -27.96 & & -5.53 & & 0.05 & \\
\hline \multicolumn{9}{|l|}{ Bienes no-duraderos } \\
\hline Nacionales (cp_nodur_bn) & -2.85 & & -5.98 & & -0.84 & & 0.51 & \\
\hline 1er Dif. (cp_nodur_bn) & -5.65 & & -56.03 & & -4.35 & & 0.04 & \\
\hline Importados (cp_nodur_bi) & -2.21 & & -3.73 & & -0.70 & & 0.51 & \\
\hline 1er Dif. (cp_nodur_bi) & -5.26 & & -46.94 & & -10.01 & & 0.06 & \\
\hline \multicolumn{9}{|l|}{$\begin{array}{l}\text { Variables explicativas } \\
\text { Índice Global de Actividades }\end{array}$} \\
\hline Económicas (igae) & -3.40 & & -7.57 & & -2.41 & & 0.20 & \\
\hline 1er Dif. (igae) & -4.20 & & -37.33 & & -2.09 & & 0.04 & \\
\hline Crédito al consumo (cre) & -2.78 & & -3.10 & & -1.12 & & 0.22 & \\
\hline 1er Dif. (cre) & -2.18 & & -11.19 & & -1.72 & & 0.35 & \\
\hline 2do Dif. (cre) & -6.88 & & -48.04 & & -4.49 & & 0.04 & \\
\hline Tipo de cambio real(tcr) & -3.22 & & -2.65 & & -0.91 & & 0.33 & \\
\hline 1er Dif. (tcr) & -5.01 & & -14.13 & & -7.89 & & 0.09 & \\
\hline
\end{tabular}

Fuente: Elaboración propia con resultados de programación con la librería urca de $\mathrm{R}$

Nota: Para las pruebas ADF, PP y KPSS se utilizaron 12 rezagos, cuatro rezagos para la prueba ERS y variables dicotómicas para incorporar factores estacionales mensuales.

Con los resultados que se presentan en el cuadro 2, de los estadísticos de las pruebas de hipótesis de cointegración $(\mathrm{r}=0)$ y de un solo vector $(\mathrm{r}<=1)$, con sus respectivos valores críticos para la traza y raíz máxima del método $J J$, se asegura que el consumo privado y sus diferentes desagregaciones están cointegrados con el ingreso corriente, el crédito y el tipo de cambio. Pero no se confirma en todos los casos un solo vector, esto ocurre únicamente para los bienes duraderos y semi-duraderos de producción nacional e importados. En el siguiente escenario, donde se relacionan los consumos con el ingreso corriente y el crédito del consumo, se constató que la mayoría de los bienes de consumo están cointegrados, pero no así los bienes duraderos de producción nacional. También se encontró que los servicios y los bienes no duraderos importados que mantenían más de una relación de largo plazo, ahora cumplen con un solo vector. Es importante recordar que la variable del crédito del consumo es de 
orden de integración 2 y al combinarse con la variable ingreso (igae) que es de $I(1)$, fue posible encontrar un vector de cointegración al considerar el problema de representación (Engle y Granger, 1987; Phillips y Hansen, 1990) y el análisis de cointegración para procesos estocásticos integrados de orden dos (Johansen, 1992; Stock y Watson, 1993). En el mismo escenario, con dos variables exógenas, pero ahora con la combinación de ingreso corriente y el tipo de cambio real, los resultados muestran que la mayoría de los bienes del consumo mantiene una relación de cointegración con un solo vector, pero en el caso de los bienes duraderos de producción nacional y los semi-duraderos importados no se encontró una relación de largo plazo. Como menciona (Mendoza-González, 2020) se ha encontrado que solo en la función del consumo privado se puede suponer como único determinante el ingreso corriente (Ruiz-Galindo y Venegas-Martínez, 2007), pero con nuestros resultados (cuadro 2) todo parece indicar que los servicios y el consumo privado cumplen con la hipótesis de cointegración con el ingreso corriente y mantener un vector que representa la relación de largo plazo; mientras que la mayoría de los bienes de consumo no están cointegrados con el ingreso corriente y, si lo están, tienen más que un solo vector de cointegración.

\subsection{Relaciones de equilibrio de largo plazo y la sensibilidad del consumo privado ante los choques de ingreso}

Del análisis de integración y de cointegración, se concluyó que el consumo privado y sus diferentes bienes y servicios mantienen relaciones económicas de largo plazo con las variables de ingreso, el crédito del consumo y el tipo de cambio real, pero existe la posibilidad de más de un vector de cointegración. En el escenario de funciones del consumo con el ingreso corriente y una de dos variables -crédito del consumo o tipo de cambio real-, se encontró que la posibilidad de relaciones de largo plazo con un solo vector de cointegración aumenta cuando se incluye el tipo de cambio real. Por último, se detectó que el consumo privado y los servicios son los únicos casos que mantienen una relación de largo plazo con el ingreso corriente y cumplen con la condición de un solo vector. Con tales condiciones de cointegración y la posibilidad de más de un vector de cointegración, se estimaron funciones consumo de largo plazo con posible sesgo de endogeneidad (Spanos, 2003) de las variables ingreso corriente, crédito del consumo y tipo de cambio real con el método de estimación FMOLS (Phillips \& Hansen, 1990; Saikkonen, 1991). Las funciones consumo de largo plazo se estimaron con diferentes combinaciones de las variables exógenas, donde la especificación sencilla incluyó al ingreso corriente sin y con constante y tendencia; en el segundo nivel de análisis, se incluyó una variable adicional en combinación con el ingreso corriente, el crédito del consumo o el tipo de cambio real; y, por último, se estimó la especificación general con las tres variables exógenas: ingreso corriente, el crédito del consumo y el tipo de cambio real. Con este procedimiento se 
contrastaron los resultados de las estimaciones, se estimó el rango de elasticidades ingreso de largo plazo y se midieron los efectos de las variables crédito y del tipo de cambio en la determinación de la sobre-sensibilidad del consumo a modificaciones del ingreso en el largo plazo.

\section{Cuadro 2}

Análisis de cointegración de Johansen y Juselius (JJ) con $V E C M$, para las funciones consumo privado y variables ingreso, crédito al consumo y tipo de cambio real

\begin{tabular}{|c|c|c|c|c|c|c|c|c|}
\hline \multirow[t]{2}{*}{ Variables } & \multicolumn{3}{|c|}{ Traza } & \multicolumn{5}{|c|}{ Raíz Máxima } \\
\hline & $\mathrm{r}=0$ & V. crítico & $\mathrm{r}<=1$ & V. crítico & $\mathrm{r}=0$ & V. crítico & $\mathrm{r}<=1$ & V. crítico \\
\hline \multicolumn{9}{|c|}{ Consumo privado agregado } \\
\hline ligae, lcre, ltcr & 124.1 & 63.0 & 60.0 & 42.4 & 64.0 & 31.5 & 37.6 & 25.5 \\
\hline ligae, lcre & 95.7 & 42.4 & 33.7 & 25.3 & 62.0 & 25.5 & 22.5 & 19.0 \\
\hline ligae, ltcr & 69.7 & 42.4 & 23.8 & 25.3 & 46.0 & 25.5 & 17.4 & 19.0 \\
\hline ligae & 52.1 & 25.3 & 11.2 & 12.3 & 40.9 & 19.0 & 11.2 & 12.3 \\
\hline \multicolumn{9}{|l|}{ Servicios } \\
\hline ligae, lcre, ltcr & 86.2 & 63.0 & 46.9 & 42.4 & 39.3 & 31.5 & 31.5 & 25.5 \\
\hline ligae, lcre & 38.6 & 42.4 & 12.9 & 25.3 & 25.7 & 25.5 & 11.5 & 19.0 \\
\hline ligae, ltcr & 49.5 & 42.4 & 18.0 & 25.3 & 31.4 & 25.5 & 14.4 & 19.0 \\
\hline ligae & 33.2 & 25.3 & 2.6 & 12.3 & 30.6 & 19.0 & 2.6 & 12.3 \\
\hline \multicolumn{9}{|c|}{ Bienes duraderos nacionales } \\
\hline ligae, lcre, ltcr & 68.9 & 63.0 & 27.0 & 42.4 & 41.9 & 31.5 & 14.6 & 25.5 \\
\hline ligae, lcre & 28.6 & 42.4 & 12.6 & 25.3 & 15.9 & 25.5 & 9.5 & 19.0 \\
\hline ligae, ltcr & 35.8 & 42.4 & 11.9 & 25.3 & 23.9 & 25.5 & 9.1 & 19.0 \\
\hline ligae & 15.6 & 25.3 & 2.8 & 12.3 & 12.8 & 19.0 & 2.8 & 12.3 \\
\hline \multicolumn{9}{|c|}{ Bienes duraderos importados } \\
\hline ligae, lcre, ltcr & 72.8 & 63.0 & 37.5 & 42.4 & 35.3 & 31.5 & 19.8 & 25.5 \\
\hline ligae, lcre & 42.7 & 42.4 & 16.4 & 25.3 & 26.3 & 25.5 & 11.3 & 19.0 \\
\hline ligae, ltcr & 44.5 & 42.4 & 21.5 & 25.3 & 23.1 & 25.5 & 15.6 & 19.0 \\
\hline ligae & 19.9 & 25.3 & 4.9 & 12.3 & 15.0 & 19.0 & 4.9 & 12.3 \\
\hline \multicolumn{9}{|c|}{ Bienes semi-duraderos nacionales } \\
\hline ligae, lcre, ltcr & 108.3 & 63.0 & 40.6 & 42.4 & 67.7 & 31.5 & 23.5 & 25.5 \\
\hline ligae, lcre & 84.4 & 42.4 & 19.6 & 25.3 & 64.8 & 25.5 & 11.1 & 19.0 \\
\hline ligae, ltcr & 57.5 & 42.4 & 22.2 & 25.3 & 35.3 & 25.5 & 16.8 & 19.0 \\
\hline ligae & 42.3 & 25.3 & 13.1 & 12.3 & 29.2 & 19.0 & 13.1 & 12.3 \\
\hline \multicolumn{9}{|c|}{ Bienes semi-duraderos importados } \\
\hline ligae, lcre, ltcr & 72.1 & 63.0 & 34.3 & 42.4 & 37.9 & 31.5 & 15.1 & 25.5 \\
\hline ligae, lcre & 45.2 & 42.4 & 18.9 & 25.3 & 26.3 & 25.5 & 13.0 & 19.0 \\
\hline ligae, ltcr & 39.1 & 42.4 & 15.6 & 25.3 & 23.5 & 25.5 & 9.4 & 19.0 \\
\hline ligae & 18.2 & 25.3 & 5.1 & 12.3 & 13.1 & 19.0 & 5.1 & 12.3 \\
\hline \multicolumn{9}{|c|}{ Bienes no-duraderos nacionales } \\
\hline ligae, lcre, ltcr & 115.4 & 63.0 & 59.1 & 42.4 & 56.2 & 31.5 & 35.6 & 25.5 \\
\hline ligae, lcre & 83.9 & 42.4 & 35.8 & 25.3 & 48.1 & 25.5 & 24.9 & 19.0 \\
\hline ligae, ltcr & 79.3 & 42.4 & 27.1 & 25.3 & 52.2 & 25.5 & 19.4 & 19.0 \\
\hline ligae & 61.9 & 25.3 & 15.4 & 12.3 & 46.5 & 19.0 & 15.4 & 12.3 \\
\hline \multicolumn{9}{|c|}{ Bienes no-duraderos importados } \\
\hline ligae, lcre, ltcr & 83.6 & 63.0 & 43.8 & 42.4 & 39.8 & 31.5 & 21.6 & 25.5 \\
\hline ligae, lcre & 65.8 & 42.4 & 27.7 & 25.3 & 38.1 & 25.5 & 18.1 & 19.0 \\
\hline ligae, ltcr & 47.8 & 42.4 & 24.6 & 25.3 & 23.2 & 25.5 & 17.7 & 19.0 \\
\hline ligae & 38.7 & 25.3 & 18.8 & 12.3 & 19.9 & 19.0 & 18.8 & 12.3 \\
\hline
\end{tabular}

Fuente: Elaboración propia con resultados de programación con la librería urca de $R$ Nota: Para todas las pruebas de traza y raíz máxima se utilizaron 12 rezagos, con tendencia lineal y variables dicotómicas para incorporar factores estacionales mensuales. 
Con la estimación de las funciones consumo sin constante y sin tendencias, encontramos un resultado que no se había analizado en trabajos previos (Mendoza-González, 2020) y que consiste en una elasticidad ingreso en el rango de 0.92 y 1.02 , que puede apoyar la hipótesis de una elasticidad del ingreso unitaria (Campbell \& Mankiw, 1989; Mallick \& Mohsin, 2016), sin importar la desagregación ni la procedencia (nacional o importada) de los bienes y servicios (cuadro 3). Esta regularidad de una elasticidad ingreso unitaria desaparece cuando en la ecuación con el ingreso corriente se incluye la constante y la tendencia -consistente con el análisis de integración y cointegración. La elasticidad ingreso del consumo privado resultó ser de 1.28 (cuadro 3), lo cual implica que la propensión marginal de largo plazo es más grande que la propensión media, en el rango entre 0.8-0.9 que indica sobresensibilidad de los consumidores a los choques de ingreso; lo que es diferente a la elasticidad unitaria estimada o condicionada en otras investigaciones (Ruiz-Galindo \& Venegas-Martínez, 2007; Galindo, 1993). También resalta que nuestra estimación de una elasticidad ingreso mayor a la unidad de largo plazo, contrasta con el mismo resultado de González- García (2002) para un periodo determinado (1989-1994), y que fue consecuencia del programa de estabilización y reforma económica a finales de 1987. De acuerdo con las investigaciones de Mendoza-González (2020), Pérez López (2012) y Carbajal De Nova y Goicoechea (2014) se ha mencionado que el consumo privado desagregado reacciona diferente a los cambios del ingreso corriente. La predicción es que los servicios son menos sensibles, y que los bienes con mayor durabilidad y de procedencia importada tienden a ser más sensibles a los choques del ingreso. Los resultados de la tabla 3 muestran que, en efecto, los servicios no son elásticos a cambios del ingreso corriente (0.36), las elasticidades ingreso de los bienes son mayores al promedio nacional (mayor a 1.28), lo cual implica que son más sensibles a los choques de ingreso, y se cumple que los bienes no duraderos como los bienes importados tienen las mayores elasticidades; las elasticidades de los bienes nacionales fluctúan entre 1.68 y 3.08 , y de los bienes importados, entre 2.74 y 8.38 .

Las especificaciones con ingreso corriente y los créditos del consumo se estimaron para analizar si, al eliminar las restricciones crediticias, se fomenta el consumo privado en general y en especial, en los bienes importados (Campbell \& Mankiw, 1989; Lavoie, 1994b; Castillo Ponce, 2003). En nuestra investigación previa (Mendoza-González, 2020), cuando el ingreso corriente y las remesas se incorporan al mismo tiempo, el efecto de los créditos del consumo reduce su importancia en la explicación tanto en el consumo privado en general, como en su desagregación en los servicios y los bienes de producción nacionales e importados. En los resultados del cuadro 3, la elasticidad del crédito del consumo fluctúa entre 0.03 y 0.18 que significa la importancia relativa en la explicación del consumo privado, en mayor medida en los bienes duraderos y principalmente importados. La combinación de 
ingreso corriente y créditos del consumo no modifican de forma relevante la elasticidad ingreso con respecto a la especificación con el ingreso corriente.

Con la segunda especificación que combina el ingreso corriente con el tipo de cambio real, lo que se espera son resultados consistentes con los que se encontraron en otras investigaciones donde las variaciones del tipo de cambio real aumentaron su importancia en la explicación de las tendencias del consumo privado y bienes importados, sobre todo con la apertura comercial (Castro, et al., 1997; Carbajal De Nova y Goicoechea, 2014). En los estudios de Campbell y Mankiw (1989) y Mallick y Mohsin (2016) se establece que el tipo de cambio o precios relativos afectan principalmente en los bienes de mayor durabilidad. En los resultados de nuestras estimaciones, se muestra que el tipo de cambio real es más importante que los créditos del consumo en la explicación del consumo privado y en especial en los bienes no-duraderos, de producción nacional e importados, aunque fueron relevantes en la explicación de los bienes duraderos importados (cuadro 3). También encontramos que en el tipo de cambio real se reduce la elasticidad ingreso corriente; de sobresensibilidad a no-elástico en el caso del consumo privado y los bienes noduraderos, y de reducción de la elasticidad ingreso de 8.38 a 4.36 en los bienes duraderos importados.

La última especificación combina las variables exógenas del ingreso corriente, los créditos del consumo y el tipo de cambio real. Los resultados encontrados son prácticamente los mismos que los dos escenarios anteriores, con el efecto pequeño de los créditos y mayor del tipo de cambio real en la explicación del consumo privado agregado, en los bienes no-duraderos nacionales $\mathrm{e}$ importados, y en los bienes duraderos importados. Con la combinación de las dos variables se observa la tendencia de la elasticidad ingreso a reducirse, de la sobre-sensibilidad a una elasticidad unitaria en el consumo privado y los bienes del consumo no-duraderos nacionales y de reducción de las elasticidades ingreso a la mitad en los bienes no-duraderos y duraderos importados; ver cuadro 3. 


\section{Cuadro 3}

Funciones de cointegración del consumo privado y su desagregación sin sesgo de endogeneidad: método de estimación $F M O L S$

\begin{tabular}{|c|c|c|c|c|c|c|c|c|c|c|}
\hline \multirow{2}{*}{$\frac{\text { Variables exógenas }}{\text { ligae }}$} & \multicolumn{5}{|c|}{ Consumo privado } & \multicolumn{5}{|c|}{ Servicios } \\
\hline & 1.00 & 1.28 & 1.26 & 0.91 & 0.98 & 0.99 & 0.36 & 0.39 & 0.49 & 0.49 \\
\hline $\operatorname{Pr}(|t|>0)$ & 0.00 & 0.00 & 0.00 & 0.00 & 0.00 & 0.00 & 0.00 & 0.00 & 0.00 & 0.00 \\
\hline lcre & & & 0.03 & & 0.04 & & & 0.01 & & 0.00 \\
\hline $\operatorname{Pr}(|t|>0)$ & & & 0.01 & & 0.00 & & & 0.40 & & 0.82 \\
\hline ltcr & & & & -0.13 & -0.15 & & & & 0.05 & 0.06 \\
\hline $\operatorname{Pr}(|t|>0)$ & & & & 0.01 & 0.00 & & & & 0.03 & 0.01 \\
\hline Constante & & -1.20 & -1.49 & 0.89 & 0.21 & & 2.59 & 2.42 & 1.83 & 1.76 \\
\hline $\operatorname{Pr}(|t|>0)$ & & 0.12 & 0.02 & 0.28 & 0.72 & & 0.00 & 0.00 & 0.00 & 0.00 \\
\hline Tendencia & & 0.00 & 0.00 & 0.00 & 0.00 & & 0.00 & 0.00 & 0.00 & 0.00 \\
\hline \multirow[t]{2}{*}{$\operatorname{Pr}(|t|>0)$} & & 0.49 & 0.05 & 0.22 & 0.57 & & 0.00 & 0.00 & 0.00 & 0.00 \\
\hline & \multicolumn{5}{|c|}{ Bienes duraderos nacionales } & \multicolumn{5}{|c|}{ Bienes duraderos importados } \\
\hline ligae & 1.00 & 3.08 & 2.49 & 2.67 & 2.49 & 0.92 & 8.38 & 7.31 & 4.36 & 4.68 \\
\hline $\operatorname{Pr}(|t|>0)$ & 0.00 & 0.00 & 0.00 & 0.00 & 0.00 & 0.00 & 0.00 & 0.00 & 0.00 & 0.00 \\
\hline lcre & & & -0.06 & & -0.07 & & & 0.11 & & 0.20 \\
\hline $\operatorname{Pr}(|t|>0)$ & & & 0.02 & & 0.02 & & & 0.23 & & 0.01 \\
\hline ltcr & & & & -0.15 & 0.00 & & & & -1.44 & -1.46 \\
\hline $\operatorname{Pr}(|t|>0)$ & & & & 0.10 & 0.99 & & & & 0.00 & 0.00 \\
\hline Constante & & -8.87 & -5.70 & -6.49 & -5.62 & & -32.28 & -28.97 & -9.09 & -12.53 \\
\hline $\operatorname{Pr}(|t|>0)$ & & 0.00 & 0.00 & 0.00 & 0.00 & & 0.00 & 0.00 & 0.04 & 0.00 \\
\hline Tendencia & & 0.00 & 0.00 & 0.00 & 0.00 & & -0.01 & -0.01 & 0.00 & 0.00 \\
\hline \multirow[t]{2}{*}{$\operatorname{Pr}(|t|>0)$} & & 0.00 & 0.03 & 0.00 & 0.05 & & 0.00 & 0.00 & 0.84 & 0.07 \\
\hline & \multicolumn{5}{|c|}{ Bienes semi-duraderos nacionales } & \multicolumn{5}{|c|}{ Bienes semi-duraderos importados } \\
\hline ligae & 1.00 & 1.74 & 1.37 & 2.10 & 1.90 & 0.92 & 2.74 & 2.44 & 1.06 & 1.26 \\
\hline $\operatorname{Pr}(|t|>0)$ & 0.00 & 0.00 & 0.00 & 0.00 & 0.00 & 0.00 & 0.00 & 0.00 & 0.02 & 0.00 \\
\hline lcre & & & -0.11 & & -0.13 & & & 0.10 & & 0.12 \\
\hline $\operatorname{Pr}(|\mathrm{t}|>0)$ & & & 0.00 & & 0.00 & & & 0.04 & & 0.00 \\
\hline ltcr & & & & 0.14 & 0.30 & & & & -0.57 & -0.63 \\
\hline $\operatorname{Pr}(|t|>0)$ & & & & 0.21 & 0.00 & & & & 0.00 & 0.00 \\
\hline Constante & & -2.98 & -0.26 & -5.12 & -3.59 & & -8.44 & -8.21 & 1.12 & -0.86 \\
\hline $\operatorname{Pr}(|t|>0)$ & & 0.05 & 0.85 & 0.01 & 0.01 & & 0.00 & 0.00 & 0.61 & 0.62 \\
\hline Tendencia & & 0.00 & 0.00 & 0.00 & 0.00 & & 0.00 & 0.00 & 0.01 & 0.00 \\
\hline \multirow[t]{2}{*}{$\operatorname{Pr}(|t|>0)$} & & 0.00 & 0.80 & 0.00 & 0.11 & & 0.08 & 0.23 & 0.00 & 0.00 \\
\hline & \multicolumn{5}{|c|}{ Bienes no-duraderos nacionales } & \multicolumn{5}{|c|}{ Bienes no-duraderos importados } \\
\hline ligae & 1.02 & 1.68 & 1.74 & 0.82 & 0.99 & 0.97 & 3.85 & 3.78 & 0.89 & 1.28 \\
\hline $\operatorname{Pr}(|t|>0)$ & 0.00 & 0.00 & 0.00 & 0.04 & 0.00 & 0.00 & 0.00 & 0.00 & 0.28 & 0.01 \\
\hline lcre & & & 0.08 & & 0.10 & & & 0.18 & & 0.24 \\
\hline $\operatorname{Pr}(|t|>0)$ & & & 0.03 & & 0.00 & & & 0.04 & & 0.00 \\
\hline ltcr & & & & -0.30 & -0.38 & & & & -1.03 & -1.21 \\
\hline $\operatorname{Pr}(|t|>0)$ & & & & 0.01 & 0.00 & & & & 0.00 & 0.00 \\
\hline Constante & & -2.67 & -3.81 & 2.24 & 0.74 & & -12.56 & -14.20 & 4.39 & 0.93 \\
\hline $\operatorname{Pr}(|t|>0)$ & & 0.15 & 0.03 & 0.25 & 0.61 & & 0.01 & 0.00 & 0.28 & 0.71 \\
\hline Tendencia & & 0.00 & 0.00 & 0.00 & 0.00 & & 0.00 & 0.00 & 0.00 & 0.00 \\
\hline $\operatorname{Pr}(|t|>0)$ & & 0.04 & 0.00 & 0.78 & 0.02 & & 0.31 & 0.04 & 0.05 & 0.99 \\
\hline
\end{tabular}

Fuente: Elaboración propia con resultados de programación con librería cointReg de $\mathrm{R}$

Nota: Todas las estimaciones fueron con constante y tendencia lineal, y variables dicotómicas para incorporar factores estacionales mensuales; para el cálculo de la varianza de largo plazo se utiliza un Bartlett kernel y la opción Andrews para el bandwidth.

\subsection{Dinámica de corto con regímenes múltiples de ajuste a equilibrios de largo por choques de ingreso}

Para identificar la dinámica de las elasticidades de corto plazo con regímenes múltiples, se aplicaron las pruebas bivariadas de Seo (2006) para la hipótesis nula de no-cointegración y alternativa cointegración-threshold, y la de Hansen- 
Seo (2002) para la hipótesis nula cointegración-lineal con respecto a la hipótesis alternativa de cointegración-threshold. Para la prueba Seo (2006), se utilizó el vector de cointegración del consumo-ingreso corriente con constante y tendencia estimada con el método FMOLS (cuadro 3). Mientras que en la prueba de Hansen-Seo (2002), se utiliza el vector de cointegración del consumo y el ingreso que fue estimado con un VECM lineal (Seo, 2011; Stigler, 2013). Los resultados de las estimaciones de las pruebas de Seo (2006), como se esperaba, indican que se acepta la hipótesis nula de no-cointegración con respecto a la hipótesis alternativa de cointegración-threshold, que es una regularidad que existe (cuadro 4). Sin embargo, con las pruebas de cointegración $J J$ y la estimación $F M O L S$ se garantiza la existencia de vectores de cointegración con el supuesto de dinámica simétrica de elasticidades ingreso de corto plazo. Los resultados de la prueba Hansen-Seo (2002), sobre el supuesto de elasticidades ingreso de corto plazo con dinámica simétrica con respecto a las asimétricas, muestran evidencia de cointegración-threshold o elasticidades ingreso de corto plazo con dinámica asimétrica para el consumo privado, servicios, bienes semi-duraderos y no-duraderos; como las probabilidades asociadas al estadístico sup-LM fueron mayor a 0.10 , no existe evidencia de comportamientos asimétricos en el caso de los bienes duraderos nacionales e importados.

Las consideraciones que se definieron en los escenarios para estimar los parámetros de ajuste al equilibrio de largo plazo, los parámetros betas y el análisis de los comportamientos asimétricos de las elasticidades de corto plazo, fueron: 1) los modelos threshold-VECM (TVECM) bivariados que utilizamos, suponen relaciones de cointegración conocidas, por eso, para el escenario básico, utilizamos las elasticidades ingreso de largo plazo del consumo solo en función del ingreso corriente, estimadas con el método FMOLS; por ejemplo, la elasticidad ingreso corriente para el consumo privado fue 1.28 , que es el resultado de la segunda columna del cuadro $3 ; 2$ ) en el mismo escenario básico, se supone que las asimetrías se derivan para diferenciar los desequilibrios por debajo y por arriba como regímenes de la función consumo de largo plazo (bajo $\left(\gamma_{L}\right)$ y alto $\left(\gamma_{H}\right)$ ), y se estiman con dos estrategias: en la primera se utiliza el mismo TVECM y parámetros betas; $\mathrm{y}$, en la segunda estrategia, se estiman TVECM individuales por régimen, lo cual implica parámetros betas individuales para cada estimación. Por último, se supone que los comportamientos asimétricos se identifican con tres regímenes: (bajo $\left(\gamma_{L}\right)$, medio $\left(\gamma_{M}\right)$ y alto $\left(\gamma_{H}\right)$ ), que se estiman también con TVECM individuales; 3 ) para analizar cómo influye la modificación del supuesto de sobre-sensibilidad, elasticidad unitaria y no-elástico en el comportamiento asimétrico de las elasticidades de corto plazo, se utilizaron las elasticidades ingreso de largo plazo de las funciones consumos, especificadas con el crédito del consumo y con crédito-tipo de cambio real de las estimaciones FMOLS del cuadro 3. 


\section{Cuadro 4}

\section{Pruebas bivariadas Seo (2006) y Hansen-Seo (2002) de cointegración threshold-VECM}

\begin{tabular}{|c|c|c|c|c|c|}
\hline \multirow[b]{2}{*}{$\begin{array}{l}\text { Relación consumo-ingreso } \\
\text { corriente }\end{array}$} & \multicolumn{2}{|c|}{$\begin{array}{l}\text { Pruebas Seo (2006): No cointegración } \\
\text { versus contegración threshold }\end{array}$} & \multicolumn{3}{|c|}{$\begin{array}{c}\text { Pruebas Hansen y Seo (2002): } \\
\text { cointegración lineal versus } \\
\text { contegración threshold }\end{array}$} \\
\hline & $\begin{array}{l}\text { Estadístico } \\
\text { sup-Wald }\end{array}$ & $\begin{array}{l}\text { P. Value }(100 \\
\text { bootstrap) }\end{array}$ & $\begin{array}{l}\text { Estadístico } \\
\text { sup-LM }\end{array}$ & $\begin{array}{l}\text { P. Value (100 } \\
\text { bootstrap) }\end{array}$ & $\begin{array}{l}\text { Max- } \\
\text { threshold } \\
\text { value }\end{array}$ \\
\hline \multicolumn{6}{|l|}{ Consumo privado } \\
\hline Total (cpt) & 19.04 & 1.00 & 71.94 & 0.03 & 0.00 \\
\hline \multicolumn{6}{|l|}{ Bienes duraderos } \\
\hline Nacionales (cp_dur_bn) & 15.75 & 1.00 & 64.35 & 0.29 & -0.03 \\
\hline Importados (cp_dur_bi) & 15.06 & 1.00 & 66.12 & 0.17 & -0.61 \\
\hline \multicolumn{6}{|l|}{ Bienes semi-duraderos } \\
\hline Nacionales (cp_semidur_bn) & 29.64 & 0.97 & 80.67 & 0.00 & 0.02 \\
\hline Importados (cp_semidur_bi) & 22.89 & 1.00 & 78.64 & 0.00 & -0.47 \\
\hline \multicolumn{6}{|l|}{ Bienes no-duraderos } \\
\hline Nacionales (cp_nodur_bn) & 19.12 & 1.00 & 88.98 & 0.00 & 0.09 \\
\hline Importados (cp_nodur_bi) & 11.49 & 1.00 & 72.68 & 0.07 & -0.08 \\
\hline
\end{tabular}

Fuente: Elaboración propia con resultados de programación con librería TsDyn de $R$

Nota: En la prueba Seo (2006) se aplica un trimming de 10\% y el vector de cointegración consumo-ingreso de la estimación FMOLS de la tabla 3; la prueba Hansen-Seo (2002) se aplicó con constante y se utiliza el vector de cointegración del VECM lineal y variables dicotómicas para incorporar factores estacionales mensuales.

Para el análisis del consumo privado, se utilizó la elasticidad ingreso de largo plazo 1.28 que supone una propensión marginal a consumir mayor que la propensión media y con valores entre 0.8 y 0.9 que indican posibles riesgos en las decisiones de largo plazo a choques de ingreso. Los resultados del escenario, con dos regímenes estimados con el mismo modelo TVECM, muestran parámetros de ajuste al equilibrio $\left(\right.$ bajo $\left(\gamma_{L}\right)$ y alto $\left.\left(\gamma_{H}\right)\right)$ con signo negativo, significativos y numéricamente muy parecidos $(-0.21$ y -0.22$)$; la correlación entre el consumo y el ingreso corriente con rezago $\left(\beta_{1}\right)$ es negativa, pequeña y no significativa; con la combinación de la elasticidad ingreso de largo plazo, los parámetros de ajuste al equilibrio y los coeficientes betas, se estimaron elasticidades ingreso de corto plazo con signo positivo muy parecidas para los regímenes, de 0.22 y 0.23 , respectivamente (cuadro 4). Los resultados del escenario, con dos regímenes y diferentes TVECM muestran un régimen bajo con signo negativo $\gamma_{L}=-0.19$ y significativo, una correlación $\beta_{1}$ negativa, con nivel de confianza bajo y una elasticidad ingreso de corto plazo negativa pero muy pequeña, que prácticamente es cero y con una tendencia con aceleramiento hacia el largo plazo. Un régimen alto con $\gamma_{H}$ positivo y significativo, $\beta_{1}$ positivo, pero no significativo, una elasticidad ingreso de corto plazo negativa, pero que no converge a la elasticidad ingreso de largo plazo con choques de ingreso. Con el escenario de tres regímenes, se confirman los resultados del régimen alto del escenario anterior, con prácticamente los mismos parámetros estimados; mientras que los escenario con bajo y medio tienen el mismo parámetro de ajuste $\gamma_{L}=\gamma_{M}=-0.17$, 
diferentes parámetros betas con los cuales se estimaron las elasticidades ingreso de corto plazo de -0.67 en régimen bajo y 0.12 en el medio. En el escenario con créditos del consumo, la elasticidad ingreso de largo plazo cambia de 1.28 a 1.26, los resultados de las estimaciones de los modelos TVECM muestran modificaciones importantes, donde los parámetros de ajuste al equilibrio (bajo $\left(\gamma_{L}\right)$ y alto $\left(\gamma_{H}\right)$ ) resultaron no significativos y cambiaron de signo. La modificación de la elasticidad ingreso por los créditos del consumo mantienen, por un lado, los resultados en el marco de dos regímenes y diferentes TVECM, y por el otro, la relevancia del régimen bajo en el modelo de tres regímenes y diferentes TVECM. Cuando el tipo de cambio real se incorpora junto a los créditos del consumo y el ingreso corriente, el escenario con el régimen bajo predomina, principalmente cuando se suponen las betas individuales con regímenes estimados con diferentes TVECM. Las efectos del ingreso sobre los servicios en el largo plazo utilizadas en los modelos TVECM resultaron no-elásticos, en todos los casos: 0.36 con el ingreso corriente; 0.39 con la variable adicional créditos del consumo; $y$, de 0.49 con las tres variables exógenas: ingreso corriente, créditos del consumo y el tipo de cambio real; ver cuadro 3. Los resultados presentados del cuadro 4 muestran que los parámetros de ajuste al equilibrio (bajo $\left(\gamma_{L}\right)$ y alto $\left(\gamma_{H}\right)$ ) son idénticos $(-0.16)$, la beta es positiva y las elasticidades ingreso de corto plazo mantienen los signos positivos, son no-elásticas y menores a la de largo plazo, con dos regímenes y un TVECM. Los resultados en todos los escenarios aplicados a los servicios, al igual que el consumo privado agregado, muestran la prevalencia del régimen bajo, pero con elasticidades de corto plazo con signos positivos.

Los bienes duraderos son los más sensibles a los choques de ingreso en el largo plazo, en especial los bienes importados tienen las elasticidades ingreso más altas en los escenarios con las tres variables exógenas: del ingreso corriente, créditos del consumo y el tipo de cambio real. Para el escenario básico, las elasticidades ingreso de los bienes duraderos nacionales e importados resultaron de 3.08 y 8.38 (ver cuadros 3 y 6), pero los parámetros de ajuste fueron ligeramente mayores, las elasticidades ingreso de corto plazo, con signo positivo y muy cercanas a la unidad, en los bienes nacionales y cero en los bienes importados. En el escenario con dos regímenes estimados con diferentes TVECM, los resultados muestran una tendencia hacia la prevalencia del escenario bajo, con parámetros de ajuste con equilibrios altos y elasticidades ingreso de corto plazo, con signo positivo y cercanos a la unidad para los bienes nacionales, y resultados inestables en el escenario alto, para los dos tipos de bienes. Los resultados son inconsistentes cuando se suponen tres regímenes debido a que los parámetros de ajuste a equilibrio son positivos, nosignificativos o en términos absolutos mayor a uno, por lo que no resulta relevante este escenario para los bienes duraderos, nacionales e importados (ver cuadro 6). 


\section{Cuadro 5 \\ Modelos bivariantes con regímenes múltiples estimados con threshold-VECM. Funciones consumo privado y servicios}

\begin{tabular}{|c|c|c|c|c|c|c|}
\hline \multirow{2}{*}{ Funciones consumo } & \multicolumn{2}{|c|}{ Sin variable exógena } & \multicolumn{2}{|c|}{ Crédito del consumo } & \multicolumn{2}{|c|}{$\begin{array}{c}\text { Crédito del consumo y tipo de } \\
\text { cambio real }\end{array}$} \\
\hline & $\begin{array}{l}\text { Consumo } \\
\text { privado }\end{array}$ & Servicios & $\begin{array}{l}\text { Consumo } \\
\text { privado }\end{array}$ & Servicios & $\begin{array}{l}\text { Consumo } \\
\text { privado }\end{array}$ & Servicios \\
\hline & \multicolumn{6}{|c|}{ Modelos con dos regímenes en ajuste de equilibrio e idéntico VECM } \\
\hline V. de coint: $(1, \mathrm{~b})$ & $(1,-1.28)$ & $(1,-0.36)$ & $(1,-1.26)$ & $(1,-0.39)$ & $(1,-0.98)$ & $(1,-0.49)$ \\
\hline $\operatorname{VC}(\gamma$ bajo $(\mathrm{L}))$ & -0.21 & -0.16 & 0.04 & -0.06 & -0.05 & -0.16 \\
\hline P. Value & 0.00 & 0.00 & 0.58 & 0.18 & 0.39 & 0.00 \\
\hline $\mathrm{VC}(\gamma$ alto $(\mathrm{H}))$ & -0.22 & -0.16 & 0.05 & -0.06 & -0.10 & -0.16 \\
\hline P. Value & 0.00 & 0.00 & 0.50 & 0.18 & 0.03 & 0.00 \\
\hline$\beta_{1}$ & -0.05 & 0.04 & -0.07 & 0.08 & -0.07 & 0.04 \\
\hline P. Value & 0.72 & 0.27 & 0.60 & 0.05 & 0.58 & 0.40 \\
\hline Elast. corto plazo (L) & 0.22 & 0.10 & -0.12 & 0.10 & -0.02 & 0.11 \\
\hline Elast. corto plazo $(\mathrm{H})$ & 0.23 & 0.10 & -0.13 & 0.10 & $\mathbf{0 . 0 3}$ & 0.11 \\
\hline \multirow[t]{2}{*}{$\begin{array}{l}\text { Porcentaje por } \\
\text { régimen }\end{array}$} & $0.81,0.18$ & $0.87,0.12$ & $0.47,0.52$ & $0.14,0.85$ & $0.69,0.30$ & $0.87,0.12$ \\
\hline & \multicolumn{6}{|c|}{ Modelos con dos regimenes en ajuste de equilibrio y diferentes VECM } \\
\hline V. de coint: $(1, \mathrm{~b})$ & $(1,-1.28)$ & $(1,-0.36)$ & $(1,-1.26)$ & $(1,-0.39)$ & $(1,-0.98)$ & $(1,-0.49)$ \\
\hline $\operatorname{VC}(\gamma$ bajo $(\mathrm{L}))$ & -0.19 & -0.23 & -0.19 & -0.17 & -0.14 & -0.11 \\
\hline P. Value & 0.03 & 0.00 & 0.02 & 0.09 & 0.03 & 0.19 \\
\hline$\beta_{1}$ & -0.25 & -0.12 & -0.27 & -0.12 & -0.42 & -0.11 \\
\hline P. Value & 0.12 & 0.03 & 0.08 & 0.06 & 0.00 & 0.07 \\
\hline Elast. corto plazo (L) & -0.01 & -0.03 & -0.03 & -0.06 & -0.29 & -0.06 \\
\hline $\mathrm{VC}(\gamma$ alto $(\mathrm{H}))$ & 0.30 & -0.04 & 0.35 & -0.06 & -0.15 & -0.07 \\
\hline P. Value & 0.06 & 0.51 & 0.08 & 0.23 & 0.59 & 0.21 \\
\hline$\beta_{1}$ & 0.17 & 0.16 & 0.30 & 0.16 & 0.47 & 0.16 \\
\hline P. Value & 0.45 & 0.02 & 0.24 & 0.01 & 0.05 & 0.01 \\
\hline Elast. corto plazo $(\mathrm{H})$ & -0.21 & 0.17 & -0.14 & 0.18 & 0.62 & 0.20 \\
\hline \multirow[t]{2}{*}{$\begin{array}{l}\begin{array}{l}\text { Porcentaje por } \\
\text { régimen }\end{array} \\
\end{array}$} & $0.63,0.36$ & $0.63,0.36$ & $0.66,0.33$ & $0.53,0.46$ & $0.7,0.3$ & $0.55,0.44$ \\
\hline & \multicolumn{6}{|c|}{ Modelos con tres regímenes en ajuste de equilibrio y diferentes $V E C M$} \\
\hline V. de coint: $(1, \mathrm{~b})$ & $(1,-1.28)$ & $(1,-0.36)$ & $(1,-1.26)$ & $(1,-0.39)$ & $(1,-0.98)$ & $(1,-0.49)$ \\
\hline $\mathrm{VC}(\gamma$ bajo $(\mathrm{L}))$ & -0.17 & -0.22 & -0.19 & -0.20 & -0.13 & -0.11 \\
\hline P. Value & 0.62 & 0.00 & 0.01 & 0.00 & 0.02 & 0.14 \\
\hline$\beta_{1}$ & -0.89 & -0.14 & -0.27 & -0.13 & -0.41 & -0.11 \\
\hline P. Value & 0.06 & 0.01 & 0.07 & 0.01 & 0.00 & 0.05 \\
\hline Elast. corto plazo (L) & -0.67 & -0.06 & -0.03 & -0.05 & -0.28 & -0.06 \\
\hline $\operatorname{VC}(\gamma$ medio $(\mathrm{M}))$ & -0.17 & -8.87 & 0.58 & -15.21 & -6.45 & 0.07 \\
\hline P. Value & 0.37 & 0.00 & 0.38 & 0.01 & 0.15 & 0.93 \\
\hline$\beta_{1}$ & -0.10 & 0.87 & 0.39 & 0.07 & 2.20 & -0.96 \\
\hline P. Value & 0.64 & 0.03 & 0.28 & 0.90 & 0.02 & 0.04 \\
\hline Elast. corto plazo (M) & 0.12 & 4.07 & -0.34 & 6.01 & 8.52 & -0.99 \\
\hline $\mathrm{VC}(\gamma$ alto $(\mathrm{H}))$ & 0.30 & -0.14 & 0.55 & -0.14 & 0.51 & -0.02 \\
\hline P. Value & 0.06 & 0.04 & 0.19 & 0.04 & 0.40 & 0.78 \\
\hline$\beta_{1}$ & 0.17 & -0.01 & -0.17 & -0.02 & -0.26 & 0.16 \\
\hline P. Value & 0.44 & 0.87 & 0.79 & 0.83 & 0.57 & 0.03 \\
\hline Elast. corto plazo $(\mathrm{H})$ & -0.21 & 0.04 & -0.87 & 0.04 & -0.76 & 0.17 \\
\hline $\begin{array}{l}\text { Porcentaje por } \\
\text { régimen }\end{array}$ & $0.26,0.36,0.36$ & $\begin{array}{l}0.57,0.11 \\
0.31\end{array}$ & $0.66,0.18,0.15$ & $\begin{array}{l}0.58,0.10 \\
0.31\end{array}$ & $0.70,0.10,0.18$ & $\begin{array}{c}0.55,0.10 \\
0.33\end{array}$ \\
\hline
\end{tabular}

Fuente: Elaboración propia con resultados de programación con librería TsDyn de $R$.

Nota: En la especificación de los threshold-VECM se aplica un trimming de 5\%, 12 rezagos, 100 bootstrap, constante, variables dicotómicas para incorporar factores estacionales mensuales y se utilizan las elasticidades ingreso de los vectores de cointegración de los modelos estimados con FMOLS del cuadro 3. 


\section{Cuadro 6 \\ Modelos bivariantes con regímenes múltiples estimados con threshold- VECM}

Funciones consumo de bienes duraderos (CBD) nacionales e importados

\begin{tabular}{|c|c|c|c|c|c|c|}
\hline \multirow{2}{*}{ Funciones consumo } & \multicolumn{2}{|c|}{ Sin variable exógena } & \multicolumn{2}{|c|}{ Crédito del consumo } & \multicolumn{2}{|c|}{$\begin{array}{c}\text { Crédito del consumo y tipo } \\
\text { de cambio real }\end{array}$} \\
\hline & $\begin{array}{c}\text { CBD } \\
\text { nacionales }\end{array}$ & $\begin{array}{c}\text { CBD } \\
\text { importados }\end{array}$ & $\begin{array}{c}\text { CBD } \\
\text { nacionales }\end{array}$ & $\begin{array}{c}\text { CBD } \\
\text { importados }\end{array}$ & $\begin{array}{c}\text { CBD } \\
\text { nacionales }\end{array}$ & $\begin{array}{c}\text { CBD } \\
\text { importados }\end{array}$ \\
\hline & \multicolumn{6}{|c|}{ Modelos con dos regímenes en ajuste de equilibrio e idéntico VECM } \\
\hline V. de coint:(1, b) & $(1,-3.08)$ & $(1,-8.38)$ & $(1,-2.49)$ & $(1,-7.31)$ & $(1,-2.67)$ & $(1,-4.68)$ \\
\hline $\mathrm{VC}(\gamma$ bajo $(\mathrm{L}))$ & -0.17 & -0.11 & -0.19 & -0.15 & -0.19 & -0.02 \\
\hline P. Value & 0.05 & 0.02 & 0.02 & 0.00 & 0.02 & 0.76 \\
\hline $\mathrm{VC}(\gamma$ alto $(\mathrm{H}))$ & -0.18 & -0.11 & -0.19 & -0.16 & -0.20 & -0.02 \\
\hline P. Value & 0.04 & 0.02 & 0.02 & 0.00 & 0.02 & 0.81 \\
\hline$\beta_{1}$ & 0.42 & -0.96 & 0.39 & -1.22 & 0.40 & -1.02 \\
\hline P. Value & 0.29 & 0.20 & 0.28 & 0.08 & 0.27 & 0.10 \\
\hline Elast. corto plazo (L) & 0.95 & -0.03 & 0.86 & -0.09 & 0.92 & -0.93 \\
\hline Elast. corto plazo $(\mathrm{H})$ & 0.97 & -0.01 & 0.88 & -0.07 & 0.94 & -0.95 \\
\hline \multirow{3}{*}{$\begin{array}{l}\text { Porcentaje por } \\
\text { régimen }\end{array}$} & & & & & & \\
\hline & $0.67,0.32$ & $0.89,0.10$ & $0.69,0.30$ & $0.72,0.27$ & $0.72,0.27$ & $0.45,0.54$ \\
\hline & \multicolumn{6}{|c|}{ Modelos con dos regímenes en ajuste de equilibrio y diferentes VECM } \\
\hline V. de coint: $(1, \mathrm{~b})$ & $(1,-3.08)$ & $(1,-8.38)$ & $(1,-2.49)$ & $(1,-7.31)$ & $(1,-2.67)$ & $(1,-4.68)$ \\
\hline $\mathrm{VC}(\gamma$ bajo $(\mathrm{L}))$ & -0.44 & -0.12 & -0.51 & -0.13 & -0.51 & -0.19 \\
\hline P. Value & 0.14 & 0.01 & 0.05 & 0.00 & 0.06 & 0.05 \\
\hline$\beta_{1}$ & -0.45 & -1.17 & -0.49 & -1.06 & -0.26 & -1.89 \\
\hline P. Value & 0.62 & 0.08 & 0.56 & 0.09 & 0.76 & 0.03 \\
\hline Elast. corto plazo (L) & 0.90 & -0.13 & 0.78 & -0.09 & 1.10 & -0.98 \\
\hline $\mathrm{VC}(\gamma$ alto $(\mathrm{H}))$ & -0.10 & 0.76 & -0.19 & -1.62 & -0.16 & -0.01 \\
\hline P. Value & 0.34 & 0.55 & 0.08 & 0.16 & 0.14 & 0.92 \\
\hline$\beta_{1}$ & -0.61 & 12.03 & -0.85 & -14.11 & -0.75 & -1.60 \\
\hline P. Value & 0.23 & 0.35 & 0.08 & 0.13 & 0.13 & 0.04 \\
\hline Elast. corto plazo (H) & -0.31 & 5.62 & -0.37 & -2.27 & -0.32 & -1.56 \\
\hline Porcentaje por & & & & & & \\
\hline \multirow[t]{2}{*}{ régimen } & $0.33,0.66$ & $0.89,0.10$ & $0.31,0.68$ & $0.89,0.10$ & $0.32,0.67$ & $0.45,0.54$ \\
\hline & \multicolumn{6}{|c|}{ Modelos con tres regímenes en ajuste de equilibrio y diferentes VECM } \\
\hline V. de coint: $(1, b)$ & $(1,-3.08)$ & $(1,-8.38)$ & $(1,-2.49)$ & $(1,-7.31)$ & $(1,-2.67)$ & $(1,-4.68)$ \\
\hline $\mathrm{VC}(\gamma$ bajo $(\mathrm{L}))$ & 0.01 & -0.12 & -0.12 & -0.12 & 0.25 & -0.19 \\
\hline P. Value & 0.99 & 0.02 & 0.71 & 0.08 & 0.53 & 0.02 \\
\hline$\beta_{1}$ & 1.63 & -0.33 & 0.56 & 0.22 & 2.07 & -1.89 \\
\hline P. Value & 0.51 & 0.66 & 0.55 & 0.79 & 0.11 & 0.01 \\
\hline Elast. corto plazo (L) & 1.62 & 0.66 & 0.87 & 1.06 & 1.41 & -1.00 \\
\hline $\mathrm{VC}(\gamma \operatorname{medio}(\mathrm{M}))$ & -1.69 & -0.21 & -0.71 & -0.10 & -0.62 & 0.04 \\
\hline P. Value & 0.00 & 0.35 & 0.62 & 0.48 & 0.27 & 0.73 \\
\hline$\beta_{1}$ & -2.90 & -4.07 & -1.23 & -2.92 & 0.34 & -0.89 \\
\hline P. Value & 0.03 & 0.03 & 0.71 & 0.01 & 0.86 & 0.30 \\
\hline Elast. corto plazo (M) & 2.31 & -2.29 & 0.53 & -2.19 & 1.98 & -1.09 \\
\hline $\mathrm{VC}(\gamma$ alto $(\mathrm{H}))$ & -0.12 & 1.83 & -0.20 & -1.53 & -0.18 & -0.95 \\
\hline P. Value & 0.23 & 0.05 & 0.03 & 0.05 & 0.06 & 0.09 \\
\hline$\beta_{1}$ & -0.71 & 22.47 & -0.90 & -13.95 & -0.88 & -7.84 \\
\hline P. Value & 0.13 & 0.02 & 0.03 & 0.10 & 0.04 & 0.02 \\
\hline Elast. corto plazo $(\mathrm{H})$ & -0.36 & 7.15 & -0.40 & -2.74 & -0.40 & -3.40 \\
\hline $\begin{array}{l}\text { Porcentaje por } \\
\text { régimen }\end{array}$ & $\begin{array}{l}0.15,0.16 \\
0.68\end{array}$ & $\begin{array}{l}0.69,0.2 \\
0.10\end{array}$ & $\begin{array}{l}0.19,0.11 \\
0.69\end{array}$ & $\begin{array}{l}0.57,0.31 \\
0.10\end{array}$ & $\begin{array}{l}0.17,0.13 \\
0.68\end{array}$ & $\begin{array}{l}0.46,0.39 \\
0.14\end{array}$ \\
\hline
\end{tabular}

Fuente: Elaboración propia con resultados de programación con librería TsDyn de $R$.

Nota: En la especificación de los threshold-VECM se aplica un trimming de 5\%, 12 rezagos, 100 bootstrap, constante, variables dicotómicas para incorporar factores estacionales mensuales y se utilizan las elasticidades ingreso de los vectores de cointegración de los modelos estimados con FMOLS del cuadro 3. 


\section{Cuadro 7 \\ Modelos bivariantes con regímenes múltiples estimados con threshold- VECM}

Funciones consumo de bienes semi-duraderos (CBSMD) nacionales e importados

\begin{tabular}{|c|c|c|c|c|c|c|}
\hline \multirow{2}{*}{ Funciones consumo } & \multicolumn{2}{|c|}{ Sin variable exógena } & \multicolumn{2}{|c|}{ Crédito al consumo } & \multicolumn{2}{|c|}{$\begin{array}{l}\text { Crédito al consumo y tipo de } \\
\text { cambio real }\end{array}$} \\
\hline & $\begin{array}{l}\text { CBSMD } \\
\text { nacionales }\end{array}$ & $\begin{array}{l}\text { CBSMD } \\
\text { importados }\end{array}$ & $\begin{array}{l}\text { CBSMD } \\
\text { nacionales }\end{array}$ & $\begin{array}{l}\text { CBSMD } \\
\text { importados }\end{array}$ & $\begin{array}{c}\text { CBSMD } \\
\text { nacionales }\end{array}$ & $\begin{array}{l}\text { CBSMD } \\
\text { importados }\end{array}$ \\
\hline & \multicolumn{6}{|c|}{ Modelos con dos regímenes en ajuste de equilibrio e idéntico VECM } \\
\hline V. de coint: $(1, \mathrm{~b})$ & $(1,-1.74)$ & $(1,-2.74)$ & $(1,-1.37)$ & $(1,-2.44)$ & $(1,-1.9)$ & $(1,-1.26)$ \\
\hline $\mathrm{VC}(\gamma$ bajo $(\mathrm{L}))$ & 0.06 & -0.20 & 0.06 & $-\mathbf{0 . 2 0}$ & 0.06 & -0.17 \\
\hline P. Value & 0.21 & 0.00 & 0.27 & 0.00 & 0.21 & 0.00 \\
\hline $\mathrm{VC}(\gamma$ alto $(\mathrm{H}))$ & 0.07 & -0.21 & 0.07 & -0.21 & 0.07 & -0.21 \\
\hline P. Value & 0.17 & 0.00 & 0.20 & 0.00 & 0.17 & 0.00 \\
\hline$\beta_{1}$ & -0.72 & -1.35 & -0.69 & -1.28 & -0.70 & -1.17 \\
\hline P. Value & 0.00 & 0.00 & 0.01 & 0.00 & 0.01 & 0.00 \\
\hline Elast. corto plazo (L) & -0.83 & -0.80 & -0.77 & -0.78 & -0.83 & -0.95 \\
\hline Elast. corto plazo $(\mathrm{H})$ & -0.85 & -0.78 & -0.79 & -0.76 & -0.84 & -0.91 \\
\hline \multirow[t]{2}{*}{$\begin{array}{l}\text { Porcentaje por } \\
\text { régimen }\end{array}$} & $0.42,0.57$ & $0.19,0.80$ & $0.35,0.64$ & $0.18,0.81$ & $0.42,0.57$ & $0.21,0.78$ \\
\hline & \multicolumn{6}{|c|}{ Modelos con dos regímenes en ajuste de equilibrio y diferentes VECM } \\
\hline V. de coint: $(1, \mathrm{~b})$ & $(1,-1.74)$ & $(1,-2.74)$ & $(1,-1.37)$ & $(1,-2.44)$ & $(1,-1.9)$ & $(1,-1.26)$ \\
\hline $\mathrm{VC}(\gamma$ bajo $(\mathrm{L}))$ & 0.21 & -0.71 & 0.05 & -0.50 & 0.26 & -0.38 \\
\hline P. Value & 0.12 & 0.01 & 0.61 & 0.03 & 0.09 & 0.04 \\
\hline$\beta_{1}$ & -1.13 & -2.57 & -1.23 & -3.30 & -0.74 & -2.66 \\
\hline P. Value & 0.00 & 0.05 & 0.01 & 0.00 & 0.08 & 0.01 \\
\hline Elast. corto plazo (L) & -1.50 & -0.6 & -1.30 & -2.08 & -1.24 & -2.19 \\
\hline $\mathrm{VC}(\gamma$ alto $(\mathrm{H}))$ & 0.13 & -0.13 & 0.00 & -0.13 & 0.12 & -0.20 \\
\hline P. Value & 0.22 & 0.03 & 0.98 & 0.05 & 0.22 & 0.01 \\
\hline$\beta_{1}$ & -0.17 & -1.19 & -0.57 & -1.11 & -0.08 & -1.09 \\
\hline P. Value & 0.67 & 0.00 & 0.09 & 0.00 & 0.84 & 0.00 \\
\hline Elast. corto plazo $(\mathrm{H})$ & -0.39 & -0.84 & -0.57 & -0.81 & -0.32 & -0.84 \\
\hline \multirow[t]{2}{*}{$\begin{array}{l}\text { Porcentaje po } \\
\text { régimen }\end{array}$} & $0.42,0.57$ & $0.18,0.81$ & $0.36,0.63$ & $0.19,0.80$ & $0.39,0.60$ & $0.19,0.80$ \\
\hline & \multicolumn{6}{|c|}{ Modelos con tres regímenes en ajuste de equilibrio y diferentes VECM } \\
\hline V. de coint: $(1, b)$ & $(1,-1.74)$ & $(1,-2.74)$ & $(1,-1.37)$ & $(1,-2.44)$ & $(1,-1.9)$ & $(1,-1.26)$ \\
\hline $\mathrm{VC}(\gamma$ bajo $(\mathrm{L}))$ & -1.10 & -0.71 & -8.51 & -0.67 & -1.90 & -0.48 \\
\hline P. Value & 0.57 & 0.00 & 0.01 & 0.01 & 0.42 & 0.00 \\
\hline$\beta_{1}$ & -4.71 & -2.57 & -10.77 & -2.39 & -5.73 & -3.01 \\
\hline P. Value & 0.09 & 0.04 & 0.00 & 0.05 & 0.10 & 0.00 \\
\hline Elast. corto plazo (L) & -2.79 & -0.62 & 0.89 & -0.74 & -2.12 & -2.40 \\
\hline $\operatorname{VC}(\gamma$ medio $(\mathrm{M}))$ & -0.03 & -0.15 & -0.36 & -0.19 & -0.03 & 7.72 \\
\hline P. Value & 0.90 & 0.12 & 0.25 & 0.09 & 0.90 & 0.06 \\
\hline$\beta_{1}$ & -1.50 & -2.01 & -1.01 & -2.50 & -1.20 & 4.48 \\
\hline P. Value & 0.00 & 0.00 & 0.20 & 0.00 & 0.02 & 0.29 \\
\hline Elast. corto plazo (M) & -1.46 & -1.61 & -0.51 & -2.05 & -1.15 & -5.25 \\
\hline $\mathrm{VC}(\gamma$ alto $(\mathrm{H}))$ & 0.13 & -0.03 & -0.03 & -0.10 & 0.13 & -0.33 \\
\hline P. Value & 0.20 & 0.87 & 0.71 & 0.59 & 0.23 & 0.02 \\
\hline$\beta_{1}$ & -0.17 & -0.48 & -0.53 & -0.46 & -0.16 & -1.04 \\
\hline P. Value & 0.66 & 0.51 & 0.08 & 0.44 & 0.67 & 0.01 \\
\hline Elast. corto plazo $(\mathrm{H})$ & -0.39 & -0.39 & -0.49 & -0.21 & -0.41 & -0.63 \\
\hline $\begin{array}{l}\begin{array}{l}\text { Porcentaje por } \\
\text { régimen }\end{array} \\
\end{array}$ & $\begin{array}{c}0.10,0.31 \\
0.57\end{array}$ & $\begin{array}{c}0.18,0.48 \\
0.33\end{array}$ & $0.10,0.21,0.68$ & $\begin{array}{c}0.18,0.36 \\
0.45\end{array}$ & $0.11,0.30,0.58$ & $\begin{array}{c}0.24,0.10 \\
0.65\end{array}$ \\
\hline
\end{tabular}

Fuente: Elaboración propia con resultados de programación con librería TsDyn de $R$.

Nota: En la especificación de los threshold-VECM se aplica un trimming de 5\%, 12 rezagos, 100 bootstrap, constante, variables dicotómicas para incorporar factores estacionales mensuales y se utilizan las elasticidades ingreso de los vectores de cointegración de los modelos estimados con FMOLS del cuadro 3. 


\section{Cuadro 8}

\section{Modelos bivariantes con regímenes múltiples estimados con threshold-}

\section{VECM}

Funciones consumo de bienes no-duraderos (CBND) nacionales e importados

\begin{tabular}{|c|c|c|c|c|c|c|}
\hline \multirow{2}{*}{ Funciones consumo } & \multicolumn{2}{|c|}{ Sin variable exógena } & \multicolumn{2}{|c|}{ Crédito al consumo } & \multicolumn{2}{|c|}{$\begin{array}{l}\text { Crédito al consumo y tipo de } \\
\text { cambio real }\end{array}$} \\
\hline & $\begin{array}{c}\text { CBND } \\
\text { nacionales }\end{array}$ & $\begin{array}{c}\text { CBND } \\
\text { importados }\end{array}$ & $\begin{array}{c}\text { CBND } \\
\text { nacionales }\end{array}$ & $\begin{array}{c}\text { CBND } \\
\text { importados }\end{array}$ & $\begin{array}{c}\text { CBND } \\
\text { nacionales }\end{array}$ & $\begin{array}{l}\text { CBND } \\
\text { importados }\end{array}$ \\
\hline & \multicolumn{6}{|c|}{ Modelos con dos regímenes en ajuste de equilibrio e idéntico VECM } \\
\hline V. de coint: $(1, b)$ & $(1,-1.68)$ & $(1,-3.85)$ & $(1,-1.74)$ & $(1,-3.78)$ & $(1,-0.99)$ & $(1,-1.28)$ \\
\hline $\operatorname{VC}(\gamma$ bajo $(\mathrm{L}))$ & -0.05 & -0.04 & -0.18 & -0.09 & -0.29 & -0.12 \\
\hline P. Value & 0.16 & 0.21 & 0.00 & 0.00 & 0.00 & 0.00 \\
\hline $\operatorname{VC}(\gamma$ alto $(\mathrm{H}))$ & -0.04 & -0.04 & -0.19 & -0.09 & -0.09 & -0.16 \\
\hline P. Value & 0.25 & 0.24 & 0.00 & 0.00 & 0.00 & 0.00 \\
\hline$\beta_{1}$ & -0.13 & -0.93 & -0.30 & -0.77 & -0.17 & -0.67 \\
\hline P. Value & 0.30 & 0.00 & 0.02 & 0.01 & 0.17 & 0.02 \\
\hline Elast. corto plazo (L) & -0.06 & -0.77 & 0.02 & -0.43 & 0.12 & -0.51 \\
\hline Elast. corto plazo $(\mathrm{H})$ & -0.07 & -0.78 & 0.03 & -0.43 & -0.08 & -0.47 \\
\hline Porcentaje por & & & & & & \\
\hline \multirow[t]{2}{*}{ régimen } & $0.15,0.84$ & $0.66,0.33$ & $0.25,0.74$ & $0.90,0.09$ & $0.25,0.74$ & $0.17,0.82$ \\
\hline & \multicolumn{6}{|c|}{ Modelos con dos regímenes en ajuste de equilibrio y diferentes VECM } \\
\hline V. de coint: $(1, b)$ & $(1,-1.68)$ & $(1,-3.85)$ & $(1,-1.74)$ & $(1,-3.78)$ & $(1,-0.99)$ & $(1,-1.28)$ \\
\hline $\operatorname{VC}(\gamma$ bajo $(\mathrm{L}))$ & 0.57 & $\mathbf{- 0 . 0 7}$ & -0.05 & -0.09 & -0.19 & -1.24 \\
\hline P. Value & 0.25 & 0.16 & 0.42 & 0.06 & 0.23 & 0.05 \\
\hline$\beta_{1}$ & -0.58 & -1.04 & -0.42 & -1.18 & -0.88 & -4.54 \\
\hline P. Value & 0.54 & 0.05 & 0.06 & 0.01 & 0.01 & 0.00 \\
\hline Elast. corto plazo (L) & -1.54 & -0.76 & -0.34 & -0.82 & -0.69 & -2.96 \\
\hline $\operatorname{VC}(\gamma$ alto $(\mathrm{H}))$ & -0.03 & -0.11 & -0.10 & -0.14 & -0.04 & -0.16 \\
\hline P. Value & 0.43 & 0.13 & 0.26 & 0.07 & 0.34 & 0.00 \\
\hline$\beta_{1}$ & 0.00 & -0.95 & -0.02 & -0.85 & 0.14 & -0.65 \\
\hline P. Value & 0.98 & 0.02 & 0.94 & 0.05 & 0.31 & 0.02 \\
\hline Elast. corto plazo $(\mathrm{H})$ & 0.05 & -0.54 & 0.16 & -0.32 & 0.19 & -0.45 \\
\hline Porcentaje por & & & & & & \\
\hline \multirow[t]{2}{*}{ régimen } & $0.18,0.81$ & $0.43,0.56$ & $0.47,0.52$ & $0.48,0.51$ & $0.25,0.74$ & $0.17,0.82$ \\
\hline & \multicolumn{6}{|c|}{ Modelos con tres regímenes en ajuste de equilibrio y diferentes VECM } \\
\hline V. de coint:(1, b) & $(1,-1.68)$ & $(1,-3.85)$ & $(1,-1.74)$ & $(1,-3.78)$ & $(1,-0.99)$ & $(1,-1.28)$ \\
\hline $\operatorname{VC}(\gamma$ bajo $(\mathrm{L}))$ & -0.34 & -0.11 & -0.06 & -0.09 & -0.20 & -1.11 \\
\hline P. Value & 0.21 & 0.29 & 0.18 & 0.04 & 0.18 & $\mathbf{0 . 0 3}$ \\
\hline$\beta_{1}$ & -1.26 & -2.76 & -0.38 & -1.23 & -0.91 & -4.24 \\
\hline P. Value & 0.04 & 0.00 & 0.06 & 0.00 & 0.01 & 0.00 \\
\hline Elast. corto plazo (L) & -0.69 & -2.35 & -0.27 & -0.88 & -0.71 & -2.82 \\
\hline $\operatorname{VC}(\gamma$ medio $(\mathrm{M}))$ & -0.14 & -0.82 & 0.05 & -0.31 & -0.10 & -0.39 \\
\hline P. Value & 0.05 & 0.09 & 0.85 & 0.05 & 0.14 & 0.08 \\
\hline$\beta_{1}$ & 0.34 & -0.25 & 0.72 & -0.66 & -0.12 & -1.44 \\
\hline P. Value & 0.07 & 0.84 & 0.04 & 0.25 & 0.51 & 0.05 \\
\hline Elast. corto plazo (M) & 0.57 & 2.90 & 0.64 & 0.50 & -0.02 & -0.94 \\
\hline $\mathrm{VC}(\gamma$ alto $(\mathrm{H}))$ & -0.13 & -0.11 & 0.03 & 0.08 & -0.04 & -0.13 \\
\hline P. Value & 0.62 & 0.10 & 0.90 & 0.87 & 0.90 & 0.22 \\
\hline$\beta_{1}$ & -0.41 & -0.81 & -0.64 & 0.67 & 0.29 & -0.46 \\
\hline P. Value & 0.28 & 0.03 & 0.10 & 0.62 & 0.34 & 0.19 \\
\hline Elast. corto plazo $(\mathrm{H})$ & -0.20 & -0.39 & -0.69 & 0.38 & 0.33 & -0.30 \\
\hline $\begin{array}{l}\begin{array}{l}\text { Porcentaje por } \\
\text { régimen }\end{array} \\
\end{array}$ & $0.24,0.52,0.23$ & $\begin{array}{l}0.28,0.15 \\
0.56\end{array}$ & $0.49,0.27,0.23$ & $\begin{array}{l}0.51,0.35 \\
0.13\end{array}$ & $0.25,0.51,0.23$ & $\begin{array}{c}0.20,0.26 \\
0.53\end{array}$ \\
\hline
\end{tabular}

Fuente: Elaboración propia con resultados de programación con librería TsDyn de $R$.

Nota: En la especificación de los threshold-VECM se aplica un trimming de 5\%, 12 rezagos, 100 bootstrap, constante, variables dicotómicas para incorporar factores estacionales mensuales y se utilizan las elasticidades ingreso de los vectores de cointegración de los modelos estimados con FMOLS del cuadro 3.

Los modelos bivariantes threshold-VECM que fueron estimados para los bienes semi-duraderos dieron resultados contrastantes. En primer lugar, las 
elasticidades de largo plazo muestran que estos bienes se parecen al comportamiento de los bienes duraderos. Esto es, las elasticidades ingreso de los bienes nacionales son mayores a la unidad (entre 1.3 y 1.9), y tienen un rango menor de efectos que los bienes importados (entre son 1.3 y 2.7), pero en los dos casos existen sobre-sensibilidad a los cambios de ingreso (ver cuadro 7). En el escenario básico, consumo en función de ingreso corriente, los resultados de los modelos TVECM que fueron aplicados a los bienes semiduraderos nacionales, indican que los parámetros de ajuste en equilibrio no fueron significativos para ningún tipo de regímenes incluyendo los escenarios con ingreso corriente y créditos al consumo, y las mismas variables y el tipo de cambio real. En cambio, en los bienes semi-duraderos importados, los parámetros de ajuste al equilibrio son muy parecidos: cuando se suponen dos regímenes estimados con un TVECM, predomina el régimen bajo, cuando se utilizan TVECM individuales y parámetros de ajuste al equilibrio menores, cuando se incorporan las variables de créditos del consumo y el tipo de cambio en las funciones consumo. En los dos tipos de bienes semi-duraderos, nacionales e importados, predominan elasticidades ingreso de corto plazo con signo negativo y tienden a ser mayores en los bienes nacionales con el escenario básico y en los modelos para los bienes importados, cuando se incorpora el tipo de cambio real (cuadro 7).

Los bienes no-duraderos nacionales son los segundos más importantes después de los servicios -representan el 33\% del consumo privado- $y$, entre los bienes no duraderos y duraderos importados, aportan el ocho por ciento. Aunque la importancia de los bienes no-duraderos de producción nacional ha disminuido en el periodo de análisis, las elasticidades ingreso de largo plazo muestran un comportamiento muy parecido al consumo privado; 1.68 cuando se considera el ingreso corriente únicamente, ligeramente mayor cuando se incluyen los créditos del consumo y unitaria cuando se añade el tipo de cambio real; ver cuadros 3 y 8 . Mientras que las elasticidades ingreso de largo plazo de los bienes no-duraderos muestran la sobre-sensibilidad, en la funciones con el ingreso corriente y el crédito al consumo $(3.85$ y 3.78$)$ y, aunque se reduce con el tipo de cambio real (1.28), siguen mostrando una sensibilidad alta; ver cuadros 3 y 8 . Las estimaciones de lo modelos bivariantes threshold- $V E C M$ para los bienes no-duraderos nacionales con el ingreso corriente, muestran parámetros de ajuste no significativos, con valores mayores a uno en términos absolutos en los modelos con dos regímenes, y es significativo con el signo correcto en el régimen medio cuando se suponen tres regímenes. Cuando se incluyen los créditos y sobre todo el tipo de cambio real, se observa el predominio del régimen bajo con parámetros de ajuste significativo, negativo y mayor numéricamente en comparación con los otros escenarios, donde las elasticidades de corto plazo se caracterizan por signos negativos y menores a la unidad; ver cuadro 8. Los resultados de los modelos TVECM que fueron estimados para los bienes no-duraderos importados, son similares a los 
encontrados en los bienes nacionales en los escenarios con el ingreso corriente. La diferencia se encontró en los modelos con tipo de cambio real, debido a que los parámetros de ajuste son significativos y negativos, en valor absoluto mayor a la unidad, lo cual indica problemas de estabilidad al equilibrio de largo $\mathrm{y}$ elasticidades ingreso de corto plazo, con signo negativo y mayores a la unidad, que implica la posibilidad de aumentos del consumo por el crecimiento de los precios relativos en el corto plazo (cuadro 8).

\section{Conclusiones}

Con un enfoque poskeynesiano, se prueba la existencia de la sobre-sensibilidad del consumo a choques de ingreso corriente de largo plazo, que se define como la presencia de una elasticidad ingreso mayor a la unidad en combinación con una propensión marginal a consumir mayor a 0.8 , en contraposición a la hipótesis de "sensibilidad excesiva" de la teoría del ingreso permanente. La sobre-sensibilidad del consumo de largo plazo se encuentra vinculado a los comportamientos asimétricos del consumo a choques de ingreso de corto plazo. En particular, con la aplicación del análisis de cointegración de JohasenJuselius y la estimación de las relaciones de cointegración sin sesgo de endogeneidad FMOLS, se concluye que la regularidad de una elasticidad ingreso corriente, con un valor unitario, aparece en funciones consumo, donde no es relevante el consumo autónomo, y el comportamiento del ingreso corriente es el único factor explicativo.

Cuando se considera el enfoque poskeynesiano en la explicación del consumo, los resultados econométricos muestran que la existencia de sobre-sensibilidad se encuentra explicado, principalmente, por los precios relativos o tipo de cambio real, la apertura comercial y el boom crediticio vinculado a la liberación financiera. Del análisis del consumo desagregado, se confirmó, que: los servicios son menos sensibles y los bienes con mayor durabilidad y de procedencia importada tienden a ser más sensibles a los choques del ingreso en el largo plazo. Los precios relativos o tipo de cambio real afectan principalmente a bienes no-duraderos, de producción nacional e importados, y a bienes duraderos importados.

Con la metodología de regímenes múltiples en la metodología de cointegración, se concluyó que existen comportamientos asimétricos entre el consumo y el ingreso corriente de corto plazo. Para el caso del consumo privado, los comportamientos son consistentes con un escenario de desaceleración o crisis (régimen bajo) y sobre-sensibilidad a choques de ingreso de largo plazo. También se detectaron escenarios sin sobresensibilidad, con mayor influencia de precios relativos o tipo de cambio real en el largo plazo, y con una tendencia hacia reacciones negativas del consumo a los choques de ingreso, en el corto plazo. 
Los servicios son tan sensibles como el consumo privado a comportamientos asimétricos en periodos de crisis económicas; los bienes duraderos son los más sensibles a los choques de ingreso en el largo plazo; los bienes semi-duraderos no tienen un patrón definido; $\mathrm{y}$, por último los bienes de consumo no-duraderos nacionales -los segundos más importantes después de los servicios- y los bienes no-duraderos importados, muestran relaciones de largo plazo con comportamientos similares a los bienes nacionales.

Finalmente, aunque los resultados de nuestra investigación aportan elementos relevantes a la discusión sobre la sensibilidad y asimetrías a choques de ingreso, en el consumo privado de México, es importante mencionar que desde el enfoque teórico y analítico, pueden existir sesgos por un problema de agregación en análisis macroeconómico que, en la literatura especializada, se elimina con el manejo de indicadores por persona y hogares, donde las preferencias de los consumidores se controlan por condiciones sociodemográficas. Este posible sesgo será abordado con una nueva línea de investigación, para probar las hipótesis poskeynesianas planteadas con los micro-datos disponibles, y analizar los impactos de la aplicación de instrumentos de política pública en la economía mexicana.

\section{Referencias}

[1] Arrau, P. y Oks, D., 1992. El ahorro privado en México, 1980-1990. Economía Mexicana. Nueva Época, 1(2), pp. 311-378.

[2] Bayoumi, T. y Sgherri, S., 2007. On tne impact of income and policy shocks on consumption, Amsterdam, The Netherlands: De Nederlandsche Bank, working paper, No. 152.

[3] Blundell, A., Browne, F., Cavaglia, S. y Tarditi, A., 1992. Finacial Liberalisation and Consumption Behaviour, s.l.: Economic Research Department, Reserve Bank of Australia.

[4] Bunn, P., Leroux, J., Reinold, K. y Surico, P., 2017. The consumption response to positive and negative income changes, London: Bank of England, Working Paper No. 645.

[5] Campbell, J. Y. y Mankiw, N. G., 1989. Consumption, Income, and Interest Rates: Reinterpreting the Time Series Evidence. NBER Macroeconomics Annual, Issue 4, pp. 185-216.

[6] Campos, V. R. y Meléndez, Á. M., 2013. Una estimación semiparamétrica de las pautas de consumo e ingreso a lo largo del ciclo de vida para México. El Trimestre Económico, octubre-diciembre(320), pp. 805-840.

[7] Carbajal De Nova, C. y Goicoechea, J., 2014. Consumo privado por origen y componente: efectos ingreso y precio para México. investigación económica, LXXIII(289), pp. 111-139.

[8] Castillo Ponce, R. A., 2003. Restricciones de liquidez, canal de crédito y consumo en México. Economía mexicana, XII(1), pp. 65-101. 
[9] Castro, C., Loría, E. y Mendoza, M. A., 1997. Eudoxio; Modelo Macro econométrico de la Economía Mexicana. México: Facultad de Economía, UNAM.

[10] Commault, J., 2017. How Does Consumption Respond to a Transitory Income Shock? Reconciling Natural Experiments and Structural Estimations, France: Ecole Polytechnique and CREST.

[11] De la Cruz, J. L. y Alcántara, J. Á., 2011. Crecimiento económico y el crédito bancario: un análisis de causalidad para México. Revista de Economía, XXVIII(77), pp. 13-38.

[12] Dickey, D. A. y Fuller, W. A., 1979. Distributions of the Estimators For Autoregressive Time Series with a Unit Root. Journal of the American Statistical Association, Issue 75, pp. 427-431.

[13] Dickey, D. A. y Fuller, W. A., 1981. Likelihood Ratio Statistics for Autoregressive Time Series with a Unit Root. Econometrica, Issue 49, pp. 1057-1072.

[14] Elizondo, R., 2019. Estimaciones del PIB mensual en México basadas en el IGAE. Estudios Económicos, 34(2).

[15] Elliott, G., Rothenberg, T. y Stock, J., 1996. Efficient Tests for an Autoregressive Unit Root. Econometrica, 64(4), p. 813-836.

[16] Engle, R. y Granger, C., 1987. Co-integration and Error Correction: Representation, Estimation, and Testing. Econometrica, Issue 55, pp. 251-276.

[17] Friedman, M., 1957. A Theory of Consumption Function. s.1.:Princeton: Princeton University Press.

[18] Galindo, L., 1993. Los determinantes de corto y largo plazos del consumo en México (1960-1988). Un análisis con mecanismo de corrección de errores y cointegración. Investigación Económica, 206(4), pp. 177-207.

[19] González García, J. R., 2002. La Dinámica del Consumo Privado en México. Un Análisis de Cointegración con Cambios de Régimen, México: Documentos de Investigación, Banxico.

[20] Hall, R., 1978. Stochastic implications of the life-cycle permanent income hypothesis: theory and evidence. Journal Political Economy, Issue 86, pp. 971-987.

[21] Hansen, B. E. y Seo, B., 2002. Testing for two-regime threshold cointegration in vector error-correction models. Journal of Econometrics, Issue 110, pp. 293318.

[22] Jappelli, T. y Pistaferri, L., 2010. The Consumption Response to Income Changes. Annual Review of Economics, Issue 2, pp. 479-506.

[23] Johansen, S. y Juselius, K., 1990. Maximum Likelihood Estimation and Inference on Cointegration - with Applications to the Demand for Money. Oxford Bulletin of Economics and Statistics, 52(2), pp. 169-210.

[24] Johansen, S., 1992. A Representation of Vector Autoregressive Processes Integrated of Order 2. Econometric Theory, 8(02), pp. 188-202.

[25] Kirchgässner, G. y Wolters, J., 2007. Introduction to Modern Time Series Analysis. 1er edición Ed. Berlin Heidelberg: Springer.

[26] Krueger, D. y Perri, F., 2011. How do Households Respond to Income Shocks?, Pennsylvania: CEPR and NBER.

[27] Kwiatkowski, D., Phillips, P., Schmidt, P. y Shin, Y., 1992. Testing the Null Hypothesis of Stationarity Against the Alternative of a Unit Root: How Sure Are We That Economic Time Series Have a Unit Root? Journal of Econometrics, Volumen 54, p. 159-178. 
[28] Lavoie, M., 1994. A Postkeynesian Approach to Consumer Choice. Journal of Post Keynesian Economics, 16(4), pp. 539-562.

[29] Lavoie, M., 1994b. Post Keynesian consumer theory: Potential synergies with consumer research and economic psychology. Journal of Economic Psychology, Issue 25, pp. 639-649.

[30] Ludwig, J., 2015. Is there heterogeneity in the response of consumption to income shocks?, Dortmund, Germany: Ruhr-Universität Bochum (RUB), Department of Economics, Economic Papers, No. 595.

[31] Mallick, S. K. y Mohsin, M., 2016. Macroeconomic Effects of Inflationary Shocks with Durable and Non-Durable Consumption. Open Economic Review, Issue 27, pp. 895-921.

[32] Mendoza-González, M. Á., 2020. Las remesas en el contexto de los factores explicativos del consumo privado en México, 1995-2017. Documento de trabajo, Posgrado de Economía, UNAM.

[33] Miller, J. L. C., 2013. Crédito bancario y crecimiento económico en México. Economía Informa, Issue 378, pp. 14-36.

[34] Pérez López, A., 2012. Estimaciones de las funciones consumo de bienes no durables y servicios y de bienes durables:1980.1-2001.3, México: Documento de Investigación No. 2002-08, Banxico.

[35] Phillips, P. y Hansen, B., 1990. Statistical Inference in Instrumental Variables Regression with I(1) Processes. Review of Economic Studies, Volumen 57, p. 99-125.

[36] Phillips, P. y Perron, P., 1988. Testing for a unit root in time series regression. Biometrika, 2(75), pp. 335-346.

[37] Ruiz-Galindo, L. A. y Venegas-Martínez, F., 2007. Un modelo macroeconométrico de simulación con microfundamentos para la economía mexicana. Economía Mexicana NUEVA ÉPOCA, XVI(2), pp. 165-217.

[38] Saikkonen, P., 1991. Asymptotically Efficient Estimation of Cointegrating Regressions. Econometric Theory, Volumen 7, pp. 1-21.

[39] Seo, M. H., 2011. Estimation of non linear error-correction models. Econometric Theory, 27(2), pp. 201-234.

[40] Seo, M., 2006. Bootstrap testing for the null of no cointegration in a threshold vector error correction model. Journal of Econometrics, 127(1), pp. 129-150.

[41] Spanos, A., 2003. Probability Theory and Statistical Inference: Econometric Modeling with Observational Data. 1ra edición ed. British: Cambridge University Press.

[42] Stigler, M., 2013. Threshold cointegration: overview and implementation in R., s.l.: Retrieved April, from http:// cran.r- project. org/web /packages/tsDyn/ vignettes/ ThCointOverview.pdf..

[43] Stock, J. H. y Watson, M., 1993. A simple estimator of cointegrating vectors in higher order integrated systems. Econometrica, Volumen 61, pp. 783-820.

[44] Urquiza, J., 2010. Income Asymmetries and the Permanent Income Hypothesis, Houston, University of Houston, Department of Economics. 